\title{
NUCLEAR STRUCTURE STUDIES
}

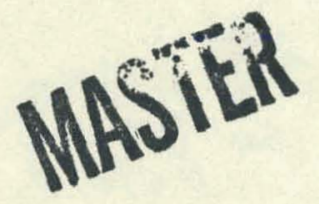

WITH

PIONS AND HEAVY IONS

\author{
Progress Report \\ for the Period June 1, 1980 - May 31, 1981
}

D. Dehnhard

University of Minnesota

Minneapolis, MN 55455

March 1981

DISCLAIMER

Prepared for

THE U.S. DEPARTMENT OF ENERGY UNDER CONTRACT

No. DOE/DE AC02-79ER10423

DISTRIBUTION OF THIS DOCUMENT IS UNLIMITED 


\section{DISCLAIMER}

This report was prepared as an account of work sponsored by an agency of the United States Government. Neither the United States Government nor any agency Thereof, nor any of their employees, makes any warranty, express or implied, or assumes any legal liability or responsibility for the accuracy, completeness, or usefulness of any information, apparatus, product, or process disclosed, or represents that its use would not infringe privately owned rights. Reference herein to any specific commercial product, process, or service by trade name, trademark, manufacturer, or otherwise does not necessarily constitute or imply its endorsement, recommendation, or favoring by the United States Government or any agency thereof. The views and opinions of authors expressed herein do not necessarily state or reflect those of the United States Government or any agency thereof. 


\section{DISCLAIMER}

Portions of this document may be illegible in electronic image products. Images are produced from the best available original document. 
Experiments performed at the Los Alamos Meson Physics Facility yielded very striking results. In inelastic pion scattering from ${ }^{14} \mathrm{C}$ near the $[3,3]$ resonance cross sections ratios $R=\sigma\left(\pi^{+}\right) / \sigma\left(\pi^{-}\right)$and $R^{-1}=\sigma\left(\pi^{-}\right) / \sigma\left(\pi^{+}\right)$were observed that are significantly larger than the free pion-nucleon values. These results were interpreted as due to strong cancellations of the neutron and proton components in the transition amplitude.

Measurements of ${ }^{13} \mathrm{C}\left(\pi, \pi^{\prime}\right)$ excitation functions between 100 and $300 \mathrm{MeV}$ at two momentum transfers showed dramatically different energy dependencies for $\Delta S=0$ and $\Delta S=1$ transitions. They were used to derive nuclear structure information on several highly excited states. Pion data for other nuclei $\left({ }^{12} \mathrm{C},{ }^{16} \mathrm{O},{ }^{40} \mathrm{Ca}\right.$, etc. $)$ were analyzed and information on isospin mixing, the double-charge-exchange reaction mechanism, and the excitation of collective states was extracted.

Further data were taken with a $547 \mathrm{MeV}$ proton beam on ${ }^{13} \mathrm{C}$. The data taking for the $\left(t, t^{\prime}\right)$ and $\left({ }^{3} \mathrm{He},{ }^{3} \mathrm{He}{ }^{\prime}\right)$ reactions at the Los Alamos Van de Graaff has been completed.

The continuing studics of the heavy-inn nucleus optical potential yielded information on very systematic dependencies of the potential depths on the incident energy. 
Table of Contents

Page

I. Introduction 1

II. Nuclear Structure Studies with Pions ' 2

1. Inelastic Scattering of $164 \mathrm{MeV}$ Pions from ${ }^{14} \mathrm{C} \quad 2$ (LAMPF Exp. \# 539).

2. Excitation Functions for Inelastic Pion Scattering from ${ }^{13} \mathrm{r}$. hetween 100 and $300 \mathrm{MeV}$ (LAMPF Exp. \# 510)

3. Elastac and Inelastic Scattcring of $\pi^{+}$aud $\|^{-}$from 1.3C (LAMPF Exp. \# 452)

4. Inelastic Scattering of $\pi^{+}$and $\pi^{-}$from ${ }^{16} \mathrm{O}$ (LAMPF Exp. \# 369 and \# 229)

5. Other Studies with Pion Beams

III. Nuclear Structure Studies with Light Ions

1. Scattering of Polarized Protons from ${ }^{13} \mathrm{C}$ at $547 \mathrm{MeV}$ (LAMPF Exp. \# 580)

2. Isospin Structure of Nuclear Excitation from a Comparison of the $\left(t, t^{\prime}\right)$ and $\left({ }^{3} \mathrm{He},{ }^{3} \mathrm{He}^{\prime}\right)$ Reactions at Low Energies

IV. Nuclear Structure Studies with Heavr Ions

1. The ${ }^{16} 0+{ }^{28}$ Si System; Energy Dependence of the Optical Fulenlid, Radial Wave Functions, ParityDependence, etc.

2. The ${ }^{16}{ }_{0}+{ }^{40} \mathrm{Ca}$ Patontinl hetwerd $\mathrm{E}_{1 \mathrm{ab}}-37$ and $65 \mathrm{MeV}$

V. Publications, Contributions to Conferences, etc.

VI. Distribution of Effort 


\section{Introduction}

This report summarizes the work done during the period from June 1, 1980 to May 31, 1981 under contract No. DEAC02-79ER10423 between the University of Minnesota and the United States Department of Energy.

The research activities involved the use of the Energetic Pion Channel and Spectrometer, EPICS, and the High Resolution Spectrometer, HRS, at the Los Alamos Meson Physics Facility. Supplementary experiments were done at the Los Alamos Van de Graaff Laboratory.

The personnel involved were the Principal Investigator, one full time Research Associate (Dr. D. B. Holtkamp) and one Graduate Student (Susan Seestrom-Morris), both at LAMPF, a part-time Research Associate (Dr. V. Shkolnik) at the University of Minnesota and an Undergraduate Student ( $J$. McBride) during the summer.

The work at EPICS was done in collaboration with H. W. Baer, R. L. Boudrie, C. L. Morris and H. A. Thiessen from Los Alamos and members of the University of Texas and the University of New Mexico users groups. The work at the Van de Graaff was done together with R. E. Brown, J. A. Cizewski, F. R. Flynn, C. L. Morris and George Idzorek from Lós Alamos and R. J. Peterson from the University of Colorado. The ( $\left.p, p^{\prime}\right)$ experiment on HRS was done together with J. F. Amann and R. L. Boudrie from Los Alamos. M. A. Franey from the University of Minnesota was involved in much of the work reported here and S. Chakravarti (also University of Minnesota) generated DWIA calculations for the pion experiments.

The pion work is summarized in section II, the light-ion work in section III, and the work on the heavy-ion optical potential in section IV. Pub1ications, contributions to conferences, etc. are listed in section $V$. 
II. Nuclear Structure Studies with Pions

1. Inelastic Scattering of $164 \mathrm{MeV}$ Pions from ${ }^{14} \mathrm{C}$ (Experiment \#539)

Differential cross sections were measured for

inelastic scattering of $164 \mathrm{MeV}^{+}$and $\pi^{-}$on ${ }^{14} \mathrm{C}$.

Large $\pi^{+} / \pi^{-}$asymmetries were observed, two being significantly larger than the free $\pi$-llucleon value.

'lhe turget consister of approximatcly 9 y uf curbon powder enriched in ${ }^{14} \mathrm{C}$ to $83 \%$. The carbon powder had been pressed into six target cells consisting of a stainless steel frame with 0.001 in. stainless steel window foils. Each target cell had an area nf $3 \mathrm{~cm} \times 3 \mathrm{~cm}$ and contained carbon of an areal density of approximatel.y $0.10 \mathrm{~g} / \mathrm{cm}^{2}$. Bccause an image of the target is created in the EPICS data analysis software it was possible to exclude the events originating from the stainless steel frames by use of suflware curs. Since the size of the ${ }^{14} \mathrm{C}$ target was less than the EPICS beam spot size (approx. $8 \mathrm{~cm} \times 20 \mathrm{~cm}$ ), strips of natural graphite were placed above and helnw the six ${ }^{14} \mathrm{C}$ target eells. Thus both ${ }^{1 ?} \mathrm{~L}$ and ${ }^{14} \mathrm{C}$ spectra could be obtained simultaneously. Additional spectra were taken from stainless steel foils as a target. The spectra from the natural graphite and the stainless steel foils enabled us to identify contaminant peaks in the ${ }^{14} \mathrm{C}$ target spectra and to derive "cleaned-up" spectra via a channel by channel subtraction (Fi,g. 1 and 2). The energy lusulution width was about $200 \mathrm{keV}$ (F.W.H.M.).

The spectra shown in Fig. 1 and 2 still include a contaminant close to the $1^{-}$state in ${ }^{14} \mathrm{C}(6.09 \mathrm{MeV})$ from ${ }^{16} \mathrm{O}\left(3^{-}, 6.13 \mathrm{MeV}\right)$ and small elastic peaks from $\mathrm{O}, \mathrm{Mg}$ and $\mathrm{Cl}$. The ${ }^{14} \mathrm{C}$ peaks were fit using the line shape of the ${ }^{12} \mathrm{C}$ 


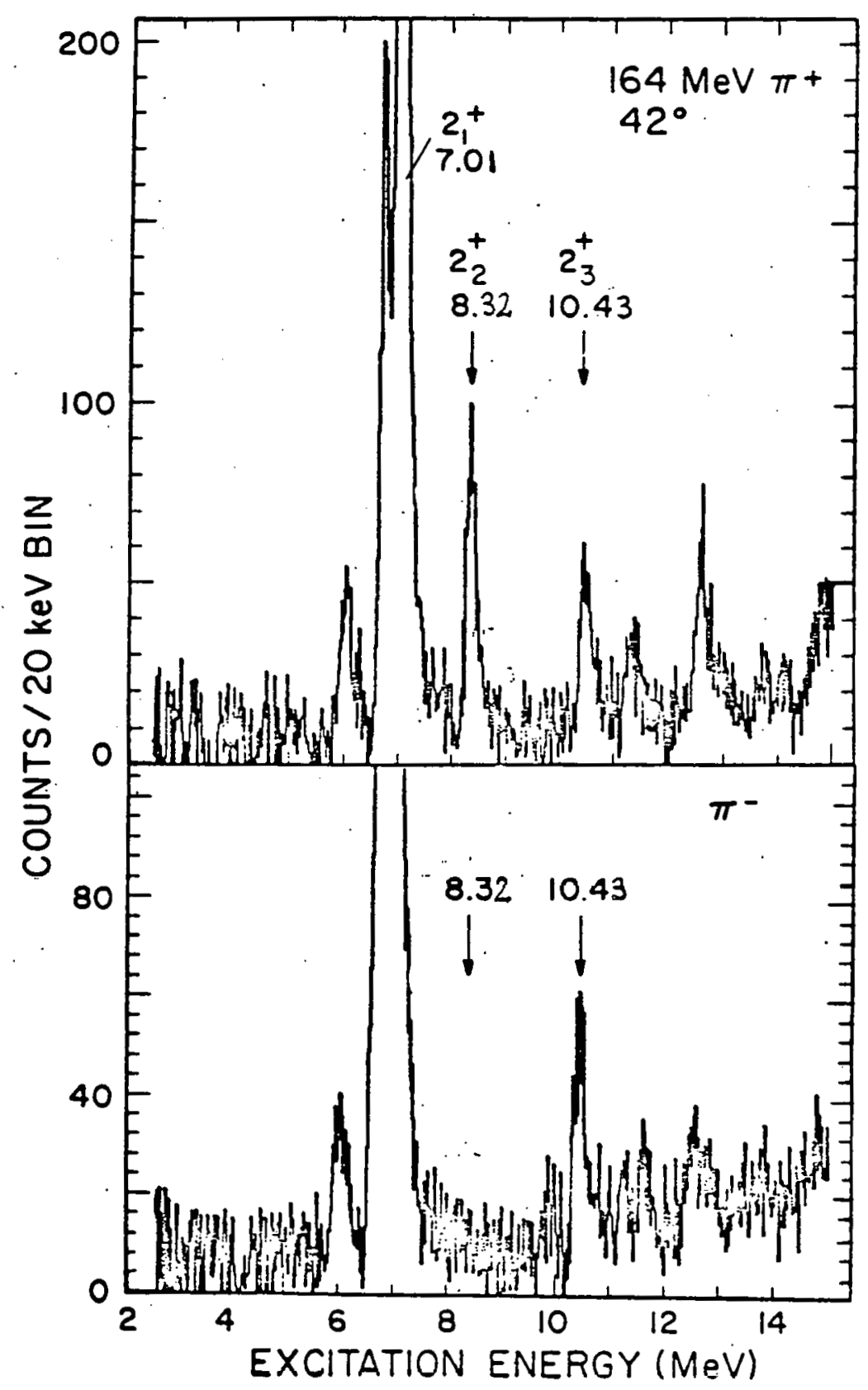

Fig. 1. Spectra frull ${ }^{14} \mathrm{C}\left(\pi, \pi^{\prime}\right)$ at $\mathrm{T}_{\pi}-164 \mathrm{McV}$ and $\theta_{1 \mathrm{ab}}=42^{\circ}$; upper spectrum from $\left(\pi^{+}, \pi^{+}\right)$, lower spectrum: $\left(\pi^{-}, \pi^{-1}\right)$. Only a fraction of the total spectrum is shown to emphasize the large asymmetry for the 2 nd $2^{+}$state at. $8.32 \mathrm{MeV}$. 


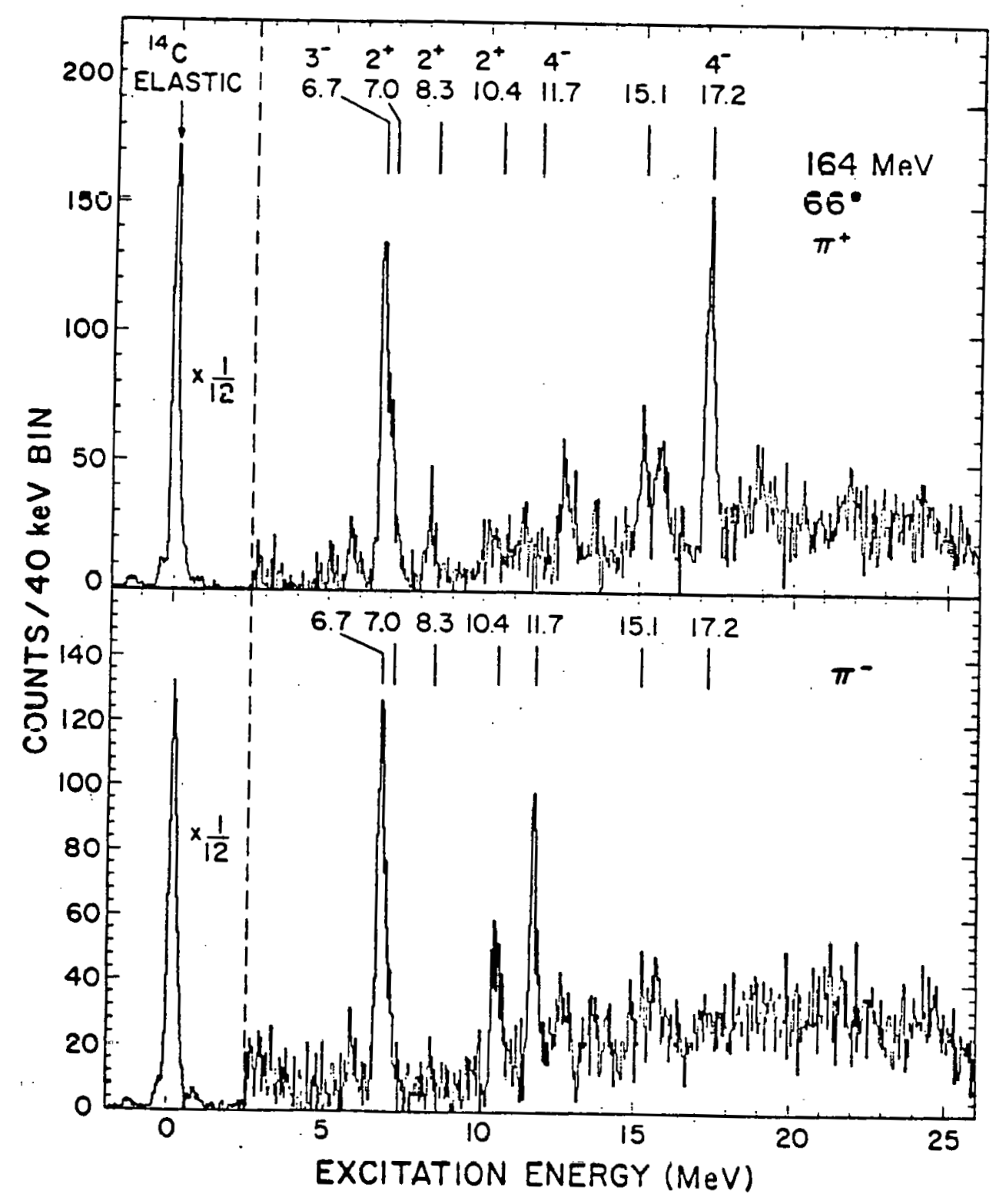

Fig. 2. Spectra from ${ }^{14} \mathrm{C}\left(\pi, \pi^{\prime}\right)$ at $\mathrm{T}_{\pi}=164 \mathrm{MeV}$ and $\theta_{1 \mathrm{ab}}=66^{\circ}$. 
(4.44 MeV) peak: For the known ${ }^{14} \mathrm{C}$ states the centroids of the peaks were kept fixed at values calculated from published excitation energies ${ }^{1}$.

The most suprising feature of these data is the sharp contrast between the near equality of $\sigma\left(\pi^{+}\right)$and $\sigma\left(\pi^{-}\right)$for the first $2^{+}$state at $7.01 \mathrm{MeV}$ and the highly asymmetric cross sections for the second $2^{+}$. state at $8.32 \mathrm{MeV}$ (Fig. 1). At a scattering angle $\left(\theta_{1 \mathrm{ab}}=42^{\circ}\right)$ which is near the first maximum of normal $\Delta \mathrm{L}=2$ angular distributions there is no evidence for a $\pi^{-}$yield for the $8.32 \mathrm{MeV}$ state. The experimental cross sections and errors yield a lower limit of $\mathrm{R}>21$ for the second $2^{+}$state with a confidence level of $90 \%$. This upper limit is significantly larger than the free $\pi$-proton value of 9 .

Similarly, at $\theta_{1 \mathrm{ab}}=66^{\circ}$ (Fig. 2), one notices among other states which exhibit $\pi^{+} / \pi^{-}$differences, a state at $11.67 \mathrm{MeV}$ which is seen essentially only in $\pi^{-}$scattering and a state at $17.23 \mathrm{MeV}$ which is strongly excited, but only in $\pi^{+}$scattering. The lower limits for the cross section ratios are $\mathrm{R}^{-1}>17$ and $\mathrm{R}>10$, respectively. Angular distributions (not shown) for these states have maxima near $66^{\circ}$, i.e. like the angular distributions for known $\Delta \mathrm{J}=4$ transitions to unnatural parity states in ${ }^{12} \mathrm{C}$ (ref. 2), $13 \mathrm{c}$ (ref. 3,4) and ${ }^{16} \mathrm{O}$. (ref. 5) which are due to excitations of the stretched $\left(\mathrm{d}_{5 / 2}, \mathrm{p}_{3 / 2}^{-1}\right)$ particle-hole configuration. On the basis of this similarity to the previous pion data we propose a $\mathrm{J}^{\pi}$ assignment of $4^{-}$for these states.

The near equality of $\sigma\left(\pi^{+}\right)$and $\sigma\left(\pi^{-}\right)$for the first $2^{+}$state and the large asymmetry for the second $2^{+}$state $\left(\sigma\left(\pi^{-}\right) \approx 0\right)$ appears to be consistent with the present knowledge of the structure of the lowest two $2^{+}$states in ${ }^{14} \mathrm{C}$. In the p-shell model calculations of Cohen and Kurath ${ }^{6}$ only one 
low lying $2^{+}$state $\left(E_{x} \approx 7 \mathrm{MeV}\right)$ is obtained and this state can only be reached by a proton excitation. However, it has been known for some time that the two lowest $2^{+}$states observed experimentally $\left(E_{x}=7.01 \mathrm{MeV}\right.$ and $8.32 \mathrm{MeV}$ ) must be very strongly configuration mixed since the p-shell strength for transfer reactions ${ }^{7}$ and the collectively enhanced electromagnetic transition ${ }^{8}$ is split between the two $2^{+}$states. Many properties of mass 14 nuclei have been explained by the weak-coupling model ${ }^{10}$ calculations of Lie ${ }^{i 1}$. For ${ }^{14} \mathrm{C}$ Lie assumed a total of seventeen $2^{+}$basis states. One of these basis states is the 2-proton hole, p-shell state of ref. 6; other basis states involve two neutrons in the $s-d$ shell and four holes in the p-shell with either the sd-particles of p-holes (or both) coupled to $2^{+}$. Lie's calculation shows nearly complete mixing between the $\left|\left(\mathrm{P}^{-2 \mathrm{P}}\right)_{2+}\right\rangle$ and $\left(\mathrm{sd}^{2 \mathrm{n}}\right)_{2+}\left(\mathrm{p}^{-4}\right)_{0+}$ basis states in the lowest two $2^{+}$states and relatively minor admixtures from the other configurations. Thus a reasonable approximation to Lie's wave functions for the lowest $2^{+}$states is

$$
\left|2_{i}^{+}\right\rangle=a_{i}\left|\left(p^{-2 p}\right)_{2+}>+\beta_{i}\right|\left(s d^{2 n}\right)_{2+}\left(p^{-4}\right)_{Q_{+}}>
$$

where $1=1,2$ refer to the first and second $2^{+}$states, respectively, and $\alpha_{1}=\alpha_{2}=\beta_{2}=\sqrt{.5}$ and $\beta_{1}=-\sqrt{.5}$. Of Lie's nine $0^{+}$basis states only two contribute significantly to the wave function of the g.s. of ${ }^{14} \mathrm{C}$ :

$$
\left|0_{1}^{+}\right\rangle=\alpha_{0}\left|\left(r^{-2 p}\right)_{U+}>+\beta_{0}\right|\left(s d^{2 n}\right)_{0+}\left(p^{-4}\right)_{0+}{ }^{\prime}
$$

We use amplitudes $\alpha_{0}=+\sqrt{.84}$ and $\beta_{0}=-\sqrt{.16}$ which are somewhat different from Lie's values but more in line with other work ${ }^{12}$. For $[3,3]$ resonance dominance and when the impulse approximation is valid $\sigma\left(\pi^{-}\right) \infty\left|3 A_{n}+A_{p}\right|^{2}$ and $\left.\sigma\left(\pi^{+}\right) \infty \mid A_{n}+3 A_{p}\right)\left.\right|^{2}$ where $A_{n}$ and $A_{p}$ are the neutron and proton components 
of the transition amplitude. If effective charges $\delta_{p}$ and $\delta_{n}$ are used to account for neglected terms in the wavefunctions we have

$$
\begin{array}{ll} 
& \sigma\left(\pi^{-}\right) \infty\left|\left(3+3 \delta_{p}+\delta_{n}\right) A_{n}+\left(1+\delta_{p}+3 \delta_{n}\right) A_{p}\right|^{2} \\
\text { and } & \sigma\left(\pi^{+}\right) \infty\left|\left(1+\delta_{p}+3 \delta_{n}\right) A_{n}+\left(3+3 \delta_{p}+\delta_{n}\right) A_{p}\right|^{2},
\end{array}
$$

(See. eq. 12 of ref. 9, extended to include both neutron and proton amplitudes.) It is clear that the opposite signs of the $\left(s d^{2}\right)$ components in the wave functions of the two $2^{+}$states will cause constructive interference between the neutron and proton contributions to the transition for one $2^{+}$state and destructive interference for the other - depending on the signs of the matrix elements of the particle-hole operator. S. Chakravarti derived these matrix elements using the weak-coupling wave functions of ref. 10 for the $\left(s d^{2}\right)$ and $\left(p^{-2}\right)$ parts of the ground and excited states in ${ }^{14} \mathrm{C}$. Preliminary DWIA calculations with the Argonne DWIA code using these matrix elements do indeed show very strong cancellation effects. Use of an effective charge $\delta_{p}=\delta_{n}=0.4$ and an $\left(s d^{2}\right)$ admlxture in the ground state of $\beta_{0}=0.4$ reproduce the experimental ratios very well but predict absolute $\sigma\left(\pi^{+}\right)$cross sections for the $2_{2}^{+}$state about a factor of two too large. Work on this problem is in progress.

The data for the two proposed $4^{-}$states at 11.67 and $17.23 \mathrm{MeV}$ (Fig. 2) are also strongly suggestive of cancellation effects. In any simple model several $4^{-}, T-1$ states are expected with large components of the stretched $\left(\mathrm{p}_{3 / 2}^{-1} \mathrm{~d}_{5 / 2}^{1}\right)$ configuration. The $4^{-}$state lowest in energy should have a large overlap with a state formed by a $d_{5 / 2}$ neutron coupled to a $3 / 2^{-}$, $\mathrm{T}=1 / 2{ }^{13} \mathrm{C}$ core (see ref. 11). This state would be reached mainly by a neutron particle-hole excitation from a $3 / 2^{-}, T=1 / 2$ core component in the ground state of ${ }^{14} \mathrm{C}$. The experimental ratio for the lower $4^{-}$state at 
$11.67 \mathrm{MeV}, \mathrm{R}^{-1}>17$, is larger than the free pion-neutron value of $\mathrm{R}^{-1} \approx 9$, indicating the presence of small contributions from proton particle-hole excitations to the transition density amplitude with a sign opposite to the one of the principal neutron particle-hole excitation.

Although the lower limit of $\mathrm{R}>10$ for the other prominent $4^{-}$state at $17.23 \mathrm{MeV}$ is consistent with a pure protion excitation $(\mathrm{R} \approx 9)$ we reject such an interpretation since it would lead to a state strongly mixed in isospin which is unlikely. A $4^{-}$state of simple $\left[p_{3 / 2}^{-1} d_{5 / 2}^{l}\right]$ structure is expected around this energy due to coupling of a $d_{5 / 2}$ nucleon to a $T=3 / 2$, mass 13 core $\left({ }^{13} \mathrm{C}\right.$ or $\left.{ }^{13_{B}}\right)$. This state would be reached from a $T=3 / 2$ core component in the g.s. by hnth proton and neutron excitations with a resulting ratio of $\mathrm{R} \approx 3$. The experimental value $\mathrm{R}>10$ implies contributions from components in the g.s. and the $4^{-}$state other than the ones mentioned above. Kurath has shown in a shell model calculation ${ }^{13}$ similar to the one of ref. 14 that the g.s. of ${ }^{14} \mathrm{C}$ does have comparable amounts of $T=1 / 2$ and $T=3 / 2$ mass 13 cores. In his model the lower $4^{-}$state (predicted at $12.7 \mathrm{MeV}$ ) is reached mainly by a neutron particle-hole cxcitation flum llue $\mathrm{T}=1 / 2$ core component in the g.s. Another $4^{-}$state (predicted at $16.9 \mathrm{MeV}$ ) is reached by a mixture of neutron and proton excitations involving both $\mathrm{T}=1 / 2$ and $\mathrm{T}=3 / 2$ cores. Cross section ratios estimated ${ }^{24}$ for these two states are in good agreement with our data for the 11.67 and $17.23 \mathrm{MeV}$ states, hut catimated abouluto cros3 sections are about a factor of two too large. 
In summary, cross section ratios larger than the free pion-nucleon values were observed for the first time in inelastic pion scattering on ${ }^{14} \mathrm{C}$ at $164 \mathrm{MeV}$. An interpretation of the results in terms of a strong cancellation of the neutron and proton components of the transition amplitudes is suggested. Thus this experiment gives a particularly convincing example for the high sensitivity of inelastic scattering of $\pi^{+}$and $\pi^{-}$to the neutron/proton structure of nuclear transitions. A manuscript is now being prepared for publication.

2. Excitation Functions for Inelastic Pion Scattering from ${ }^{13} \mathrm{C}$ between 100 and $300 \mathrm{MeV}$ (Experiment \# 510)

We have measured differential cross sections for ${ }^{13} \mathrm{C}\left(\pi, \pi^{\prime}\right)$ between 100 and $300 \mathrm{MeV}$ for momentum transfers of $1.1 \mathrm{~h} \mathrm{fm}^{-1}$ and $1.4 \mathrm{~h} \mathrm{fm}^{-1}$. In this energy range the different energy dependences of the spin-dependent alid spin-independent parts of the pion-nucleon interaction provide a very sensitive method of discriminating between transitions that proceed with a spin transfer $(\Delta S=1)$ or without a sp1n transfer $(\Delta S=0)$. Five transitions in ${ }^{13} \mathrm{C}$ were found to be dominated by this $\Delta S=1$ transition density ampli.tude.

The target was a $15 \mathrm{~cm} \times 23 \mathrm{~cm}$ sheet of carbon, $210 \mathrm{mg} / \mathrm{cm}^{2}$ thick, enriched in ${ }^{13} \mathrm{C}$ to $99 \%$, and covered on both sides by thin $\left(\approx 1 \mathrm{mg} / \mathrm{cm}^{2}\right)$ Kapton foils. The energy resolution width was about $250 \mathrm{keV}$ (FWHM). At each energy the scattering angles were chosen to keep the momentum transfer fixed at either $1.1 \mathrm{~h} \mathrm{fm}^{-1}$ or $1.4 \mathrm{~h} \mathrm{fm}^{-1}$. The former value is close to the 
first maximum in the angular distribution for a $\Delta \mathrm{L}=2$ transition and the latter is close to the maximum in the angular distribution for the strongly $\pi^{-}$enhanced transition to a state at $9.5 \mathrm{MeV}^{3,4}$.

The pion-nucleon scattering amplitude can be written in the following form if the interaction is dominated by the $[3,3]$ resonance $^{15}$ :

$$
f\left(k, k^{\prime}\right)=\alpha(k)[2 \cos (\theta)+\vec{\sigma} \cdot \hat{n} \sin (\theta)]
$$

where $k\left(k^{\prime}\right)$ is tho pion momentuiii befuse (after) the collision, $\theta$ is the scattering angle in the pion-nucleon c.m. frame, $\vec{\sigma}$ is the spin operator for the nucleon, $\hat{n}$ is the normal to the scattering plane, and the coefficient $\alpha(k)$ is energy dependent. Only thic operator $\vec{\sigma} \cdot \hat{n}$ can lead to a spin transfer $(\Delta S=1)$. Siciliano and walker have shown that a similar expression holds for pion-nucleus inelastic scattering ${ }^{16}$. In their formalism, the differential cross section for pion-nucleus inelastic scattering in the fixed scatterer approximation is given by:

$$
\alpha(\theta)-F(E)\left[4 M^{2}(q, E) \cos ^{2} \theta+s^{2}(4, E) \sin ^{2} \theta\right]
$$

assuming dominance of the $[3,3]$ resonance, the validity of the impulse approximation, and a one-step reaction mechanism. Here $E$ is the pion energy, $\mathrm{q}$ is the momentum transfer, $\theta$ is the scattering angle in the pinn-nurlelus laboratory frame, and $M(q, E)$ and $S(q, E)$ are the spin-independent and spindependent form factors, respectively. $F(E)$ contains the encrgy-dependence of the pion-nucleon amplitudes in the laboratory frame and phase space factors. The energy dependences of the form factors $M(q, E)$ and $S(q, E)$ are due to distorted wave attenuation effects and have been estimated to be very similar for values of $q=q_{o}$, i.e. near the maxima of the form factors. 
Thus this energy dependence can be factored out and combined with $F(E)$ to yield $\Gamma(E)$ so that at constant $q=q_{0}$ (ref. 16)

$$
\sigma(\theta)=\Gamma(E)\left[4 M^{2}\left(q_{0}\right) \cos ^{2} \theta+s^{2}\left(q_{0}\right) \sin ^{2} \theta\right]
$$

Both $M\left(q_{0}\right)$ and $S\left(q_{0}\right)$ contribute to natural parity transitions, but they are usually dominated by $M\left(q_{0}\right)$. Only the spin-dependent form factor can contribute to $\Delta S=1$ transitions (if Fermi motion corrections are neglected).

The scattering angle $\theta$ is a simple function of the incident energy or wave number $k$, i.e. $\theta=2 \sin ^{-1}(q / 2 k)$. To keep the momentum transfer constant at the $q$ value where the cross sections for the transitions of interest have maxima, the scattering angle needs to be decreased from about $90^{\circ}$ at $\mathrm{T}_{\pi}=100 \mathrm{MeV}$ to about $32^{\circ}$ at $\mathrm{T}_{\pi}=300 \mathrm{MeV}$. (Due to its 1 arge width the $[3,3]$ resonance is dominant over this whole energy range.) Thus the energy dependence of $\sigma(\theta)$ for $\Delta S=1$ transitions and $\Delta S=0$ transitions measured at constant momentum transfer will be very different simply due to the difference between the $\sin ^{2} \theta$ and $\cos ^{2} \theta$ functions. The energy dependences of the $\sin ^{2} \theta$ and $\cos ^{2} \Theta$ functions are in fact expected to dominate the shapes of the excitation functions since $\Gamma(E)$ depends only relatively weakly on the ellergy for constant $q$. Consequently, the cross sections for $\Delta S=1$ transitions should decrease with increasing energy since $\sin ^{2} \Theta$ decreases between $100 \mathrm{MeV}\left(\theta \approx 90^{\circ}\right)$ and $300 \mathrm{MeV}\left(\Theta \approx 32^{\circ}\right)$. In contrast, the cross sections for $\Delta S=0$ transitions should increase with increasing energy due to the associated $\cos ^{2}(\theta)$ dependence. Indeed, the work of Moore et al..$^{17}$ and Cottingame et al. ${ }^{18}$ showed that the transitions to known natural and unnatural parity states in ${ }^{12} \mathrm{C}\left(\pi, \pi^{\prime}\right)$ have dramatically different energy dependences and indicated the presence of previously unknown unnatural parity transitions. 
In the present work the $\left(\pi^{+}, \pi^{+\prime}\right)$ cross sections for the transitions to the strongly excited $3 / 2^{-}$state $(3.68 \mathrm{MeV})$, and the unresolved $5 / 2^{-}, 7 / 2^{+}$ doublet $(7.5 \mathrm{MeV})$, were measured at $\mathrm{q}=1.1 \mathrm{~h} \mathrm{fm}^{-1}$ and found to increase uniformly with energy (Fig. 3 ). The $3 / 2^{-}$and $5 / 2^{-}$states are collectively enhanced and predicted to be dominated by the $\Delta \mathrm{L}=2, \Delta S=0$ transition amplitude ${ }^{19}$. At $q=1.1 \mathrm{fm}^{-1}$ the cross sections measured for the doublet at $\% 5 \mathrm{MeV}$ are expected to be due mostly to the $5 / 2^{-}$state and Thus the excrtation function for this group should show the fcatures of a $\Delta S=0$ transition like the excitation function for the transition to the $3 / 2^{-}$state. Indeed, for both states the simple $\cos ^{2} \theta$ relation (Fig. 3 , broken lines) predicts the trend of the data quite well. A distorted wave impulse approximation (DWIA) calculation of the $3 / 2^{-}$state using the code DWPI of Eisenstein and Miller ${ }^{20}$ with a simple collective form factor reproduces the shape of the excitation function in detail (Fig. 3 , solid 1 ine). The relatively small differences between the $\cos ^{2} \Theta$ function and the DWIA result verify the claims made abovc regarding the weakness of the energy dependence of $\Gamma$.

The $\left(\pi^{-}, \pi^{-'}\right)$ excitation function for the transition to the $9.5 \mathrm{MeV}$ state was measured at $q=1.4 \hbar \mathrm{fm}^{-1}$ and found to decrease uniformly with energy (Fig. 1), in sharp contrast to the lati for the $\Delta S$ - 0 transitions discussed above. The energy dependence is very well arronunted for by $\sin ^{2}(\theta)$ (dash dotted line).

It has been proposed that this state is the $9 / 2^{+}$member of a weakcoupling multiplied ${ }^{21}$ based on a $1 d_{5 / 2}$ neutron coupled to the first exrited $2^{+}$state in ${ }^{12} \mathrm{C}$. In this model the large $\pi^{-}$asymmetry 22 $A=\left(\sigma\left(\pi^{-}\right)-\sigma\left(\pi^{+}\right) /\left(\sigma\left(\pi^{-}\right)+\sigma\left(\pi^{+}\right)\right)=-0.8 \pm 0.2\right.$ can be explained as due to a pure neutron $1 \mathrm{p}_{3 / 2} \rightarrow 1 \mathrm{~d}_{5 / 2}$ particle-hole excitation. The shell model 


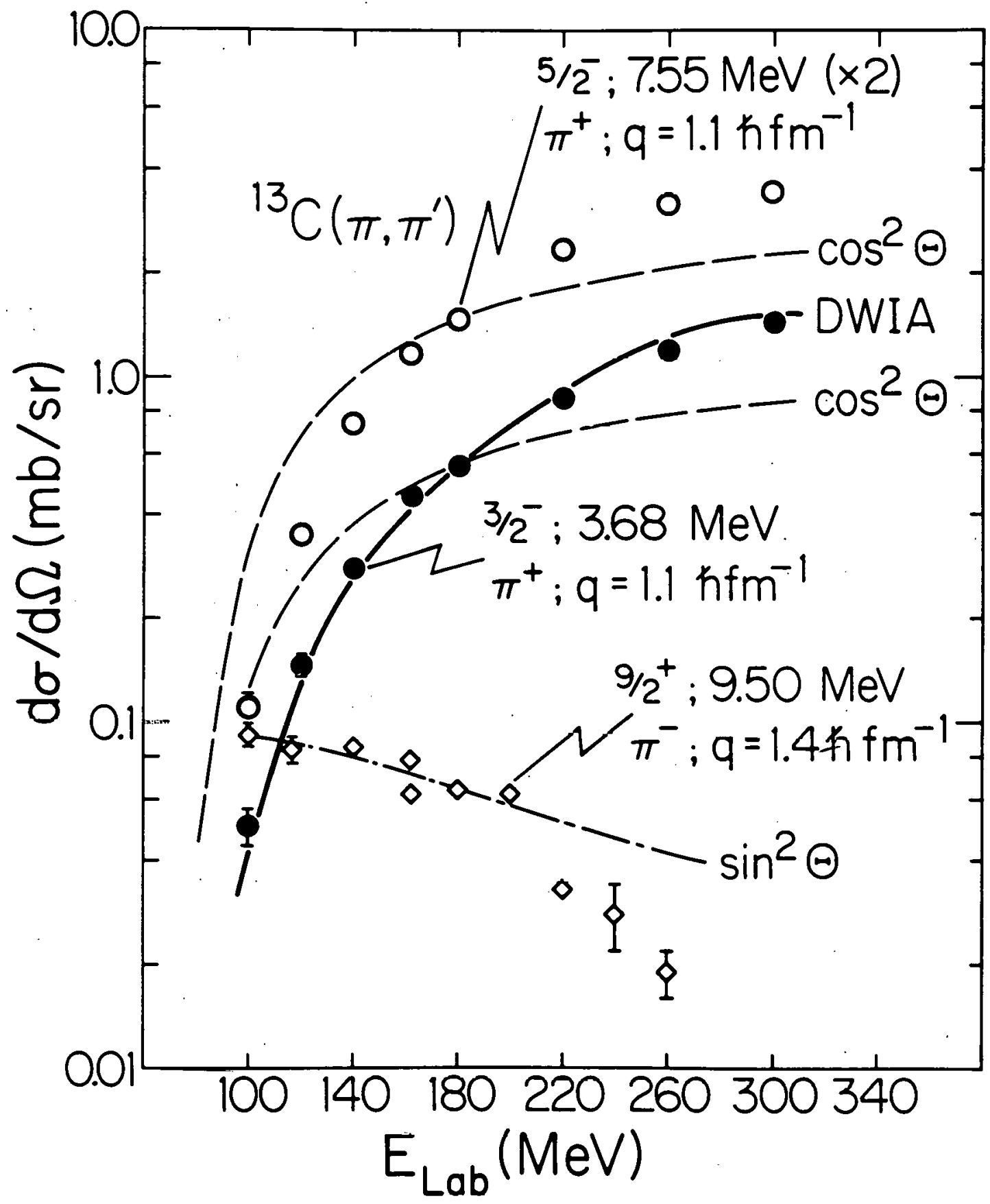

Fig. 3. Excitation functions for ${ }^{13} \mathrm{C}\left(\pi^{+}, \pi^{+'}\right)$ to the $3 / 2^{-}(3.68 \mathrm{MeV})$ and

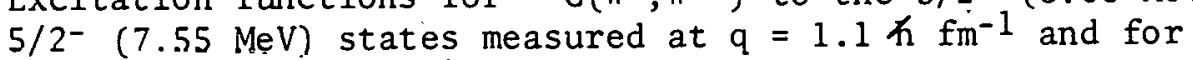
${ }_{13} \mathrm{C}\left(\pi^{-}, \pi^{-}\right)$to the $9 / 2^{+}(9.50 \mathrm{MeV})$ state measured at $\mathrm{q}=1.4 \mathrm{~h} \mathrm{fm}^{-1}$. The dashed (dash-dot) lines are the $\cos ^{2} \theta\left(\sin ^{2} \theta\right)$ dependences arbitrarily normalized to the data at $180 \mathrm{MeV}$. The solid line is the result of a DWIA calculation for the $3 / 2^{-}$state. 
calculations of Millener and Kurath ${ }^{14}$ predict a $9 / 2^{+}$state at $9.5 \mathrm{MeV}$ which has a very large overlap with the weak-coupling state. The DWIA calculations of Lee and Kurath ${ }^{4}$ using the Millener and Kurath wave functions reproduce not only the $\pi^{-}$enhancement very well but also the absolute cross sections ${ }^{3}$ for exciting this state to within about $20 \%$ According to this model the $9 / 2^{+}$state is reached solely by an unnatural parity transition with a total angular momelilum transfer $\Delta J=4$, an urbllal anguiar momentum transfer $\Delta \mathrm{L}=3$, and a spin tranfer $\Delta S=1$. The experimental excitation function for this state from the present work is characteristic of a $\Delta S=1$ transition in excellent agreement with the shell model calculation ${ }^{4,14}$.

We remark that the enhancement of $\Delta S=1$ transitions relative to $\Delta S=0$ transitions at low pion energies ( $~ 100 \mathrm{MeV}$ ) is of considerable help in searches for weak unnatural parity transitions. At or above resonance $(\gtrsim 180 \mathrm{MeV})$, such transitions may be barely visible against a background of strong $\Delta S=0$ transitions.

Four states. or grnips of statoc, in the exilabiun energy region from 12 to $24 \mathrm{McV}$ were fumid to be strongly excited at large momentum transfer. Their angular distributions are fairly structureless and peak at large angles characteristic of a large angular momentum transfer to high spin states ${ }^{3}$. The centroids of these groups are at 16.05, 17.92, 21.38 , and $21.60 \mathrm{MeV}$. Their exritation functiona at $q-1.14 \mathrm{fm}^{-1}$ are very similiar to that for the $9 / 2^{+}$state at $9.50 \mathrm{MeV}$ and they are also very well described by $\sin ^{2} \Theta$ (Fig. 4). Thus the transitions to these states must be dominated by $\Delta S=1$. The $16.05 \mathrm{MeV}$ state is $\pi^{+}$enhanced, $A=+0.6 \pm 0.2$ (ref.22), in agreement with the shell model prediction 4,14 


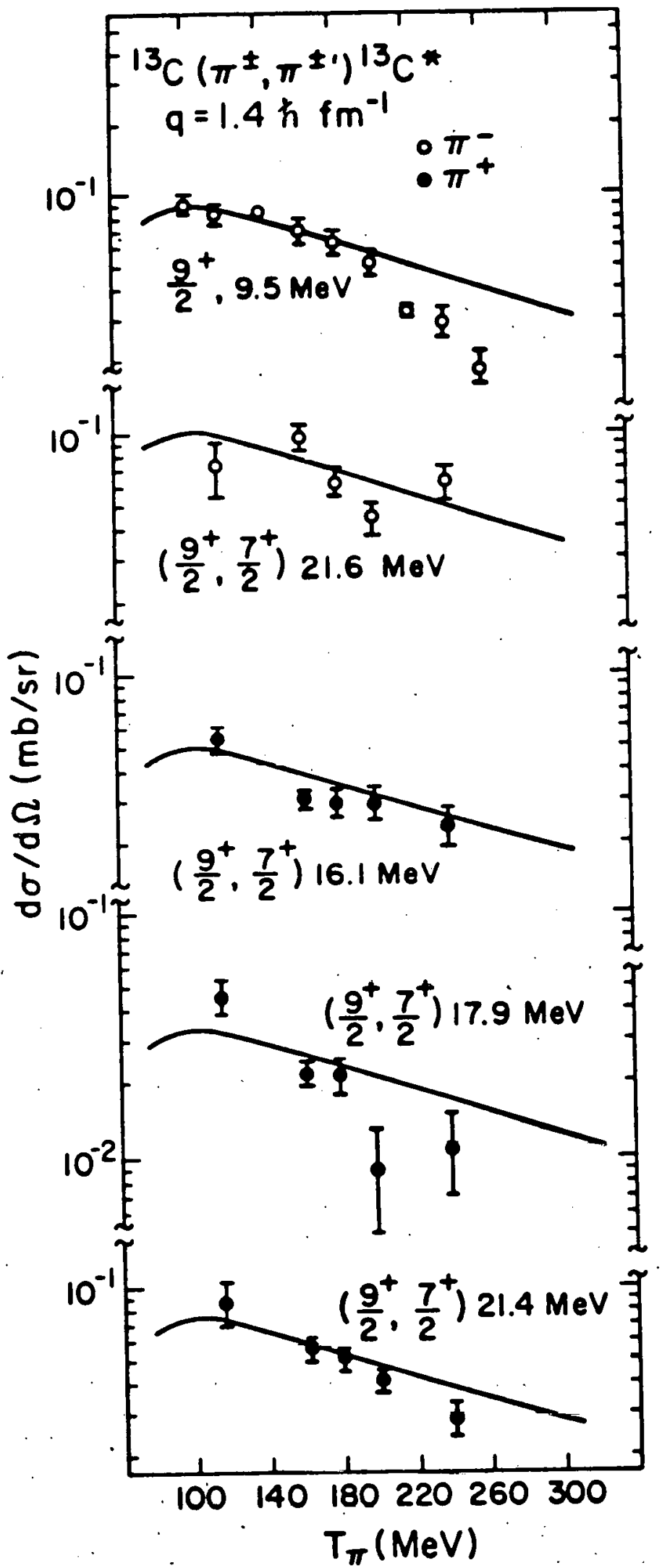

Fig. 4. Excitation functions for ${ }^{13} \mathrm{C}\left(\pi^{ \pm}, \pi^{ \pm \prime}\right)$ to states at 9.50 and 21.60 $\mathrm{MeV}$ (with $\pi^{-}$) and at $16.05,17.92$, and $21.38 \mathrm{MeV}$ (with $\pi^{+}$) measured at $q=1.4 \mathrm{~h} \mathrm{fm}^{-1}$. The solid lines are simple $\sin ^{2} \theta$ dependences. 
for a $9 / 2^{+}$state close to $16 \mathrm{MeV}$. The complex close to $21.5 \mathrm{MeV}$ peaks at $21.38 \mathrm{MeV}$ in $\pi^{+}$scattering and at $21.60 \mathrm{MeV}$ in $\pi^{-}$scattering. The state or group of states at $17.92 \mathrm{MeV}$ is excited about equally by $\pi^{+}$and $\pi^{-}$. An interpretation of these results in terms of shell model predictions is difficult because of the large number of states involved. The $21.5 \mathrm{MeV}$ complex probably contains several $7 / 2^{+}$and $9 / 2^{+}$states. Furthermore, the $T=1 / 2$ and $T=3 / 2$ states at these energies are most likely mixed in

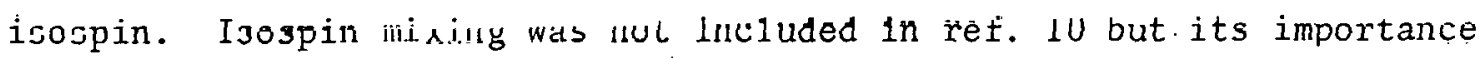
is suggested by the similarity of the pion spectra ${ }^{22}$ close to $21.5 \mathrm{MeV}$ in ${ }^{13} \mathrm{C}$ with those ${ }^{2}$ close to $19 \mathrm{MeV}$ in ${ }^{12} \mathrm{C}$.

The $180^{\circ}$ electron scattering data of rcf. 23 and 24 are in agreement with our $\Delta S=1$ assignments for some of these transitions. They found M4 transitions to states at $9.49,16.06$, and $21.43 \mathrm{MeV}$ but do not see any significant strength at $\sim 17.9 \mathrm{MeV}$. A comparison of the differcnt excitation strengths from $\left(\pi^{+}, \pi^{+\prime}\right),\left(\pi^{-}, \pi^{-1}\right)$, and $\left(e, e^{\prime}\right)$ allows conclusions about the isospin transfer $\Delta T$ since the transverse (e,e') form factor is dominated by $\Delta T=1$. The ncar equality of $\sigma\left(\pi^{-}\right)$and $\sigma\left(\pi^{+}\right)$for the group

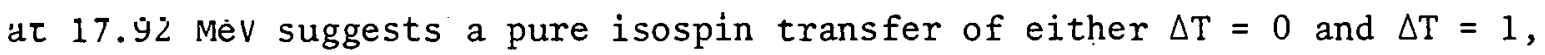
but the lack of any significant M4 strength from (e,e') strongly supports a pure $\Delta \mathrm{T}=0$ transitions. The excitation energy of one of the strong M4's from $\left(e, e^{\prime}\right), E_{x}=21.43 \pm 0.03 \mathrm{MeV}^{24}$, is close to the centroid of the peak seen in $\left(\pi^{+}, \pi^{+}\right)$. This suggesta a concentraliun of tho $\Delta T=1$ strength in the lower energy part of the $21.5 \mathrm{MeV}$ complex. A precise measurement of peak shapes and peak positions for both $\left(\pi, \pi^{\prime}\right)$, and $\left(e, e^{\prime}\right)$ would aid considerably a more detailed analys is of the $21.5 \mathrm{MeV}$ region. 
In summary, these data have demonstrated the utility of measurements of excitation functions of inelastic pion scattering in the identification of $\Delta S=1$ transitions. This technique has been used to confirm that the transition to the $9 / 2^{+}$state at $9.5 \mathrm{MeV}$ involves $\Delta S=1$ and to identify four $\Delta S=1$ transitions to states in the region from 12 to $24 \mathrm{MeV}$ in ${ }^{13} \mathrm{C}$. Knowledge of whether a transition is dominated by $\Delta S=1$ is very useful for tests of model predictions. A manuscript has been prepared recently and submitted for publication.

3. Elastic and Inelastic Scattering of $\pi^{+}$and $\pi^{-}$from ${ }^{13} \mathrm{C}$ at $162 \mathrm{MeV}$ (LNMPF Experiment \# 452)

Part of this work has been discussed in last year's report and a detailed description was recently given by Susan J. Seestrom-Morris in her Ph.D. Thesis (ref. 24). We present here only a brief discussion of some of the recent results.

\section{a. Elastic Scattering}

The finalized elastic data are presented in Fig. 5. These data were analyzed using the code DWPI of Eisenstein and Miller ${ }^{20}$ A potential of Kisslinger form was used but protons and neutrons were allowed to have different density distributions. Two sets of potential strength parameters were derived from pion-nucleon phase shifts.

We quote from ref. 3: "Set I was calculated within the code NDWPI, using the approximation of Auerbach, et a1. ${ }^{25}$ for the transformation from the pionnucleon center-of-mass system to the pion-nucleus center-of-mass system. The second set was generated using the method of Cottingame, et al. ,26 where the pion nucleon phase shifts are evaluated at an energy $30 \mathrm{MeV}$ less than the corresponding incident pion energy." Use of the second set resulted 


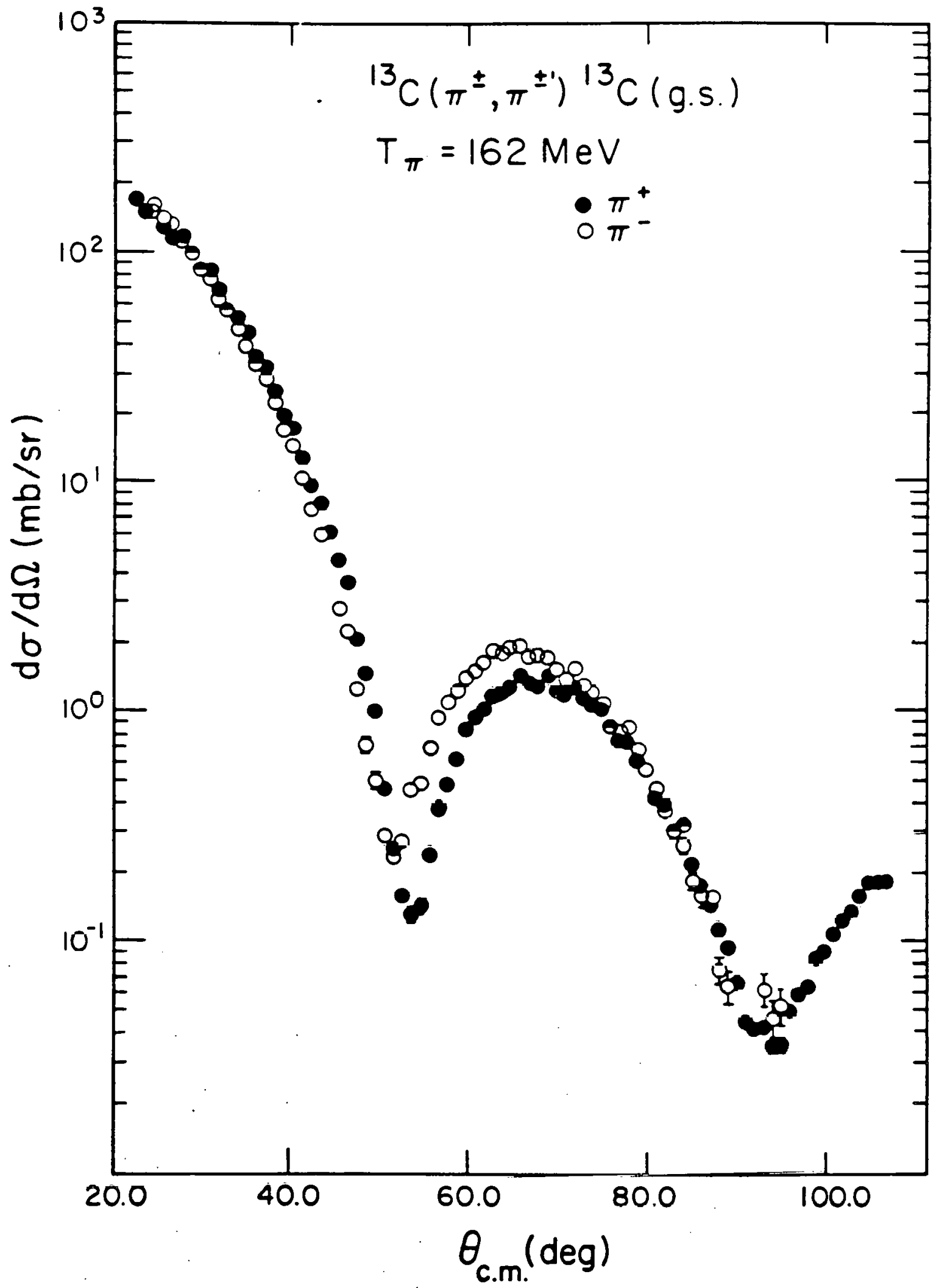

Fig. 5. Elastic scattering cross sections for ${ }^{13} \mathrm{C}+\pi^{+}$and ${ }^{13} \mathrm{C}+\pi^{-}$at $\mathrm{T}_{\pi}=162 \mathrm{MeV}$. 
in better fits to the data than use of the first (Fig.6). For the density distributions we assumed two-parameter p-shell Gaussian distributions and a three-parameter Fermi distribution (Table I ). The curves in Fig. 6 were obtained with the p-shell Gaussian labelled $\rho_{I}$ with r.m.s. radius $\left\langle r^{2}\right\rangle^{1 / 2}=2.305 \mathrm{fm}$. Fig. 7 shows the results for another p-shell Gaussian $\left.\left.\left(\rho_{\text {III }} ; \dot{\langle r}\right)^{2}\right)^{1 / 2}=2.28 \mathrm{fm}\right)$ and a three parameter Fermi distribution $\left(\rho_{\mathrm{II}}\right.$; $\left\langle\mathrm{r}^{2}\right\rangle^{1 / 2}=2.28 \mathrm{fm}$. For both calculations in Fig. 7 the "energy shifted" $V(r)$ was used. "The two densities $\rho_{I I}$ and $\rho_{\text {III }}$ produce results that agree equally well with the data out to the second maximum. The agreement is much better near the second minimum and third maximum for $\rho_{2}$ than it is for $\rho_{3}$. Since both distributions have the same rms radius, the calculations show some sensitivity to the details of the density distribution. This is probably due mostly to the difference between the radial fall-off at large radii of the two distributions. Since pions are strongly absorbed they are particularly sensitive to small differences in the surface of the nucleus.

The quality of the agreement of the calculations (done with equal r.m.s. radii for protons and neutrons) with both the $\pi^{+}$and $\pi^{-}$data shows no indication that the neutron distribution has an 'r.m.s. radius different from the proton distribution. If the neutron r.m.s. radius is increased from 2.28 to $2.35 \mathrm{fm}$ the position of the first minimum in the angular distribution is shifted by only about $1^{\circ}$. The r.m.s. radius needed to reproduce the position of the first minimum depends slightly on the energy shift used in calculating the optical potential. It seems that a systematic study of elastic scattering at many energies and from many nuclei needs to be done before reliable values can be extracted for 


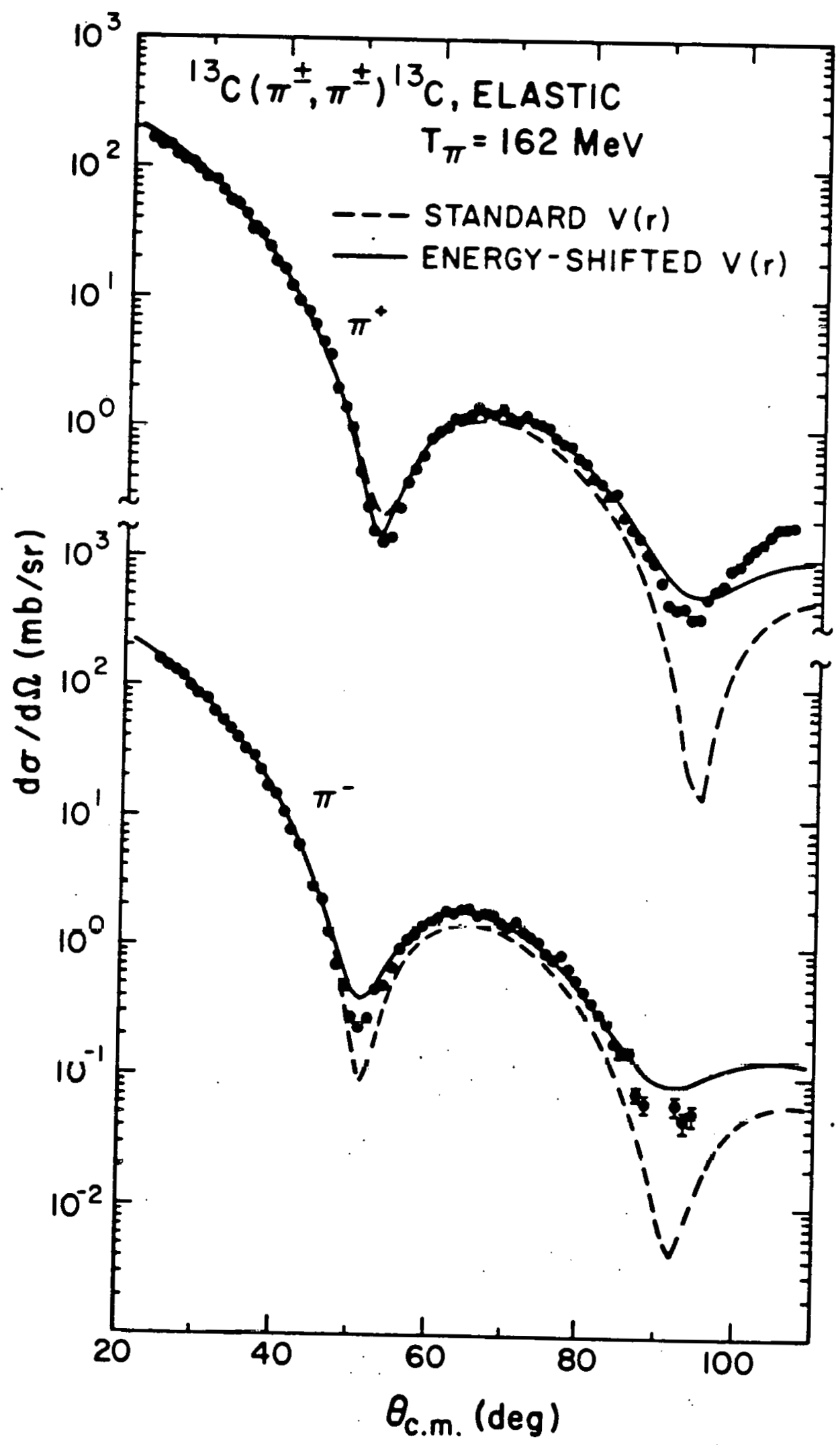

Fig. 6. Elastic data and optical model fits with a standard potential and an "energy-shifted" potential (see text) for $13 \mathrm{C}+\pi$. 


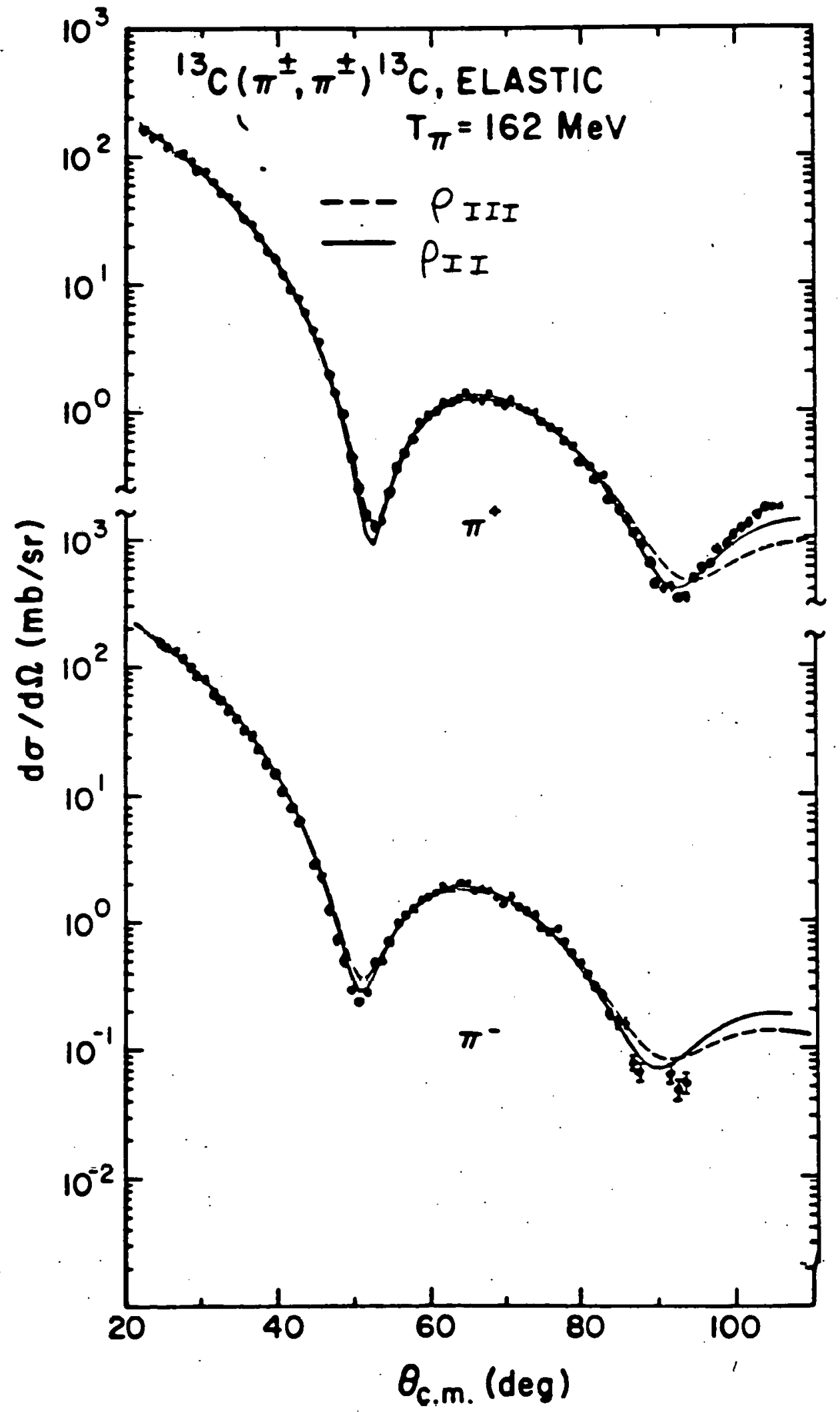

Fig. 7. Flastic data and optical model fits using two different density distributions (see text). 
Table 1 : Neutron and Proton Density Distribution Parameters

\begin{tabular}{|c|c|c|c|c|}
\hline label & $\underset{(\mathrm{fm})}{c_{\mathrm{p}}}$ & $\underset{(f m)}{c_{n}}$ & $\begin{array}{l}\left\langle\mathrm{r}^{2}\right\rangle_{\mathrm{p}}^{1 / 2} \\
(\mathrm{fm})\end{array}$ & $\begin{array}{l}<\mathrm{r}^{2}>_{\mathrm{n}}^{1 / 2} \\
(\mathrm{fm})\end{array}$ \\
\hline$p_{I}^{(a)}$ & 1.565 & 1.549 & 2.305 & 2.305 \\
\hline$\rho_{\text {II }}^{(b)}$ & 2.40 & 2.40 & 2.28 & 2.28 \\
\hline$\rho_{T T T}^{(a)}$ & 1.548 & 1.532 & 2,28 & 2.28 \\
\hline
\end{tabular}

(a) Two-parameter p-shell Gaussian distribution:

$$
\begin{aligned}
& \rho(r)=\rho_{0}\left(1+\alpha \frac{r^{2}}{c^{2}}\right) \exp \left(-\frac{r^{2}}{c^{2}}\right) \\
& \text { where } \alpha=\frac{z-2}{3} \text { for protons and } \alpha=\frac{N-2}{3} \text { for neutrons }
\end{aligned}
$$

(b) Three-parameter Fermi distribution:

$$
\begin{aligned}
& \rho(r)=\rho_{0}\left(1+\exp \frac{r-c}{z}\right)^{-1}\left(1+\frac{w r^{2}}{c^{2}}\right) \\
& \text { where } z=0.41 \mathrm{fm} \text { and } w^{\prime}=-0.16 \text { both for protons and neutrons. }
\end{aligned}
$$


the differences between the neutron and proton distributions." It also seems clear that a theoretical justification needs to be found for the energy shift method ${ }^{26}$.

We have also investigated the effect of the "inelastic contribution" to the elastic scattering. Since ${ }^{13} \mathrm{C}$ is an odd-mass nucleus, transitions can occur between, e.g., the $0 \mathrm{p}-1 \mathrm{~h}$ and $2 \mathrm{p}-1 \mathrm{~h}$ components in the ground state wavefunction. Using the transition density of Kurath ${ }^{19}$ we found only a negligibly small contribution to the elastic cross sections within the angular range covered by this experiment (DWIA calculations done by $S$. Chakravarti). 
b. Inelastic Scattering; Collective Model

A collective model analysis of the enhanced quadrupole transitions to the $3 / 2^{-}(3.68 \mathrm{MeV})$ and $5 / 2^{-}(7.55 \mathrm{MeV})$ states and the octupole transition to the $11.82 \mathrm{MeV}$ state was done. We quote from ref. 3 : "The calculations were performed with the optical potentials and transition densities (of the previous section) with a version of the rnde DWPI ${ }^{20}$, called NDWPI, which had been modified by R. Eisenstein to use separate deformation parameters, $\beta_{n}$ and $\beta_{p}$, for the neutron and proton density distributions. Calculations were first done with $\beta_{p}=\beta_{n}=1$ and then $\beta_{p}$ and $\beta_{n}$ were adjusted to reproduce the experimental peak cross sections by solving the following equations:

$$
\begin{aligned}
& R^{+}=\left(3 Z \beta_{p}+N \beta_{n}\right)^{2} /(3 Z+N)^{2} \\
& R^{-}=\left(Z \beta_{p}+3 N \beta_{n}\right)^{2} /(Z+3 N)^{2}
\end{aligned}
$$

where $\mathrm{R}^{+\cdots}$ and $\mathrm{R}^{-}$are defined by:

$$
\mathrm{R}^{ \pm}=\frac{\sigma^{ \pm}(\exp )}{\sigma^{ \pm}(D W)} \text {. }
$$

Here $\sigma^{ \pm}(D W)$ are the cross sections calculated with $\beta_{p}=\beta_{n}=1$. After $\beta_{p}$ and $B_{n}$ had been determined. from Eq.II.3.1 DWIA calculations were done with these values of $\beta_{p}$ and $\beta_{n}$ and the good fits generated with these caloulations are presented in Fig. 8 . The angular distributions for the strongly excited 11.82 MeV state are suggestive of $\Delta T_{1}=3$ and its energy is clusc to the energy of the collectively enhanced $3^{-}$state at $9.63 \mathrm{MeV}$ in ${ }^{12} \mathrm{C}$. Thus the spin of this state is suggested to be either $5 / 2^{+}$or $7 / 2^{+}$or it is a $5 / 2^{+} / 7 / 2^{+}$ doublet. The solid curves correspond to density $\rho_{\text {II }}$ and the dashed curves to density $\rho_{\text {III }}$. The quality of the agreement between the calculations and the data is 


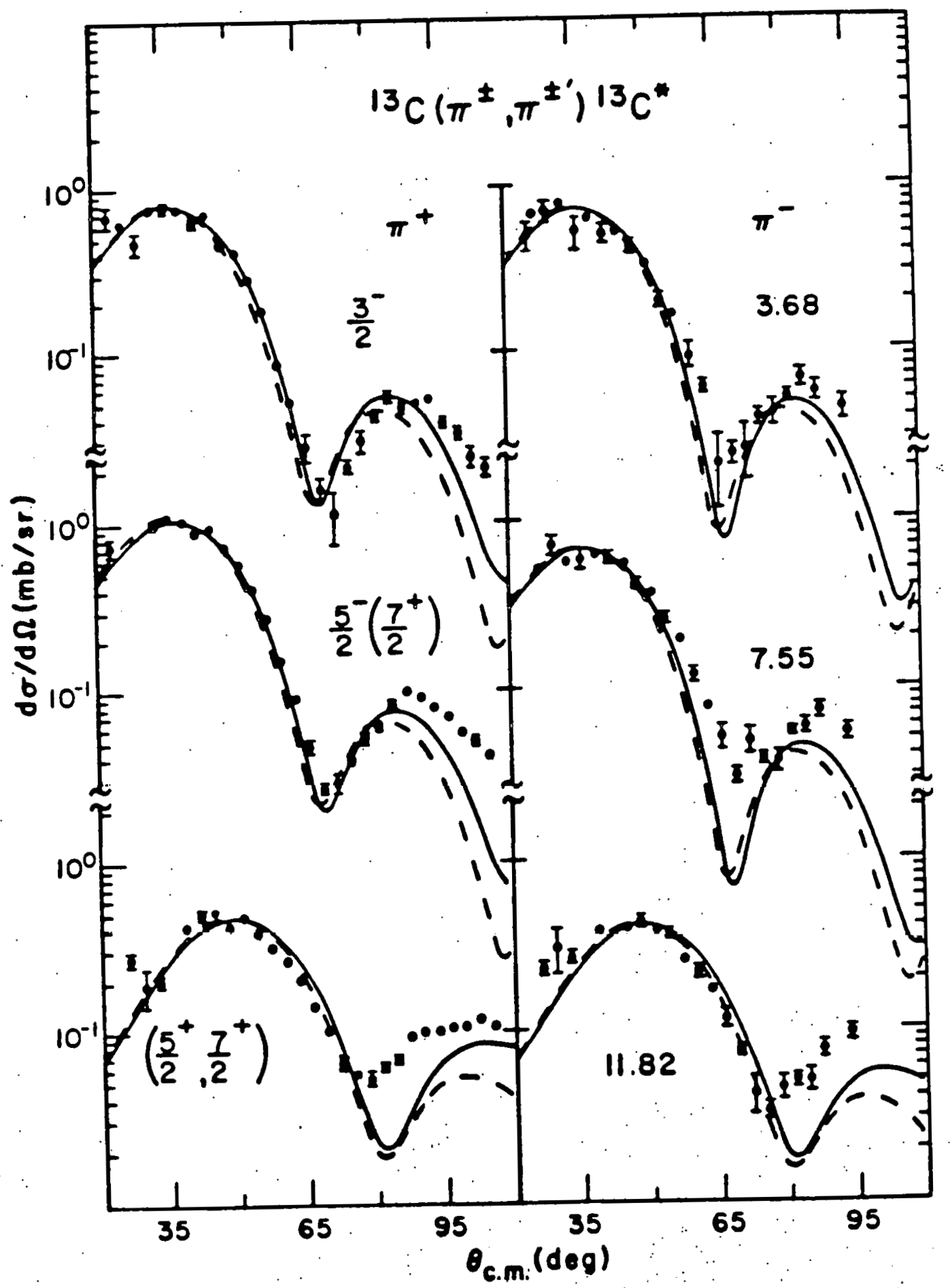

Fig. 8. Inelastic data and collective model predictions for strongly excited states in ${ }^{13} \mathrm{C}$. 
better for $\rho_{\text {III }}$ than for $\rho_{\text {II }}$ for all three states. For the $3 / 2^{-}$and $5 / 2^{-}$states, the shape of the calculations reproduces the experimental shapes quite well. For the $11.82 \mathrm{MeV}$ state the agreement is not as good, especially not at angles larger than $75^{\circ}$.

In collective model analyses of inelastic scattering it is customary to quote the deformation length, BR, obtained from the analysis. The parameter $K$ is the undeformed value of the radius parameter that is given an explicit angular dependence. However, the interpretation of the "doformation lcngths" obtained in terms of static or dynamic deformations of the nuclear shape can bc misleading. In cases, such as the $5 / 2^{-}$state, where the proton and neutron deformation lengths were found to be very different it would imply strongly different shapes for the neutron and proton distributions in the nucleus. This is not at all reasonable. The justification for the use of this model is that it provides a very simple procedure to generate a semirealistic, surface-peaked transition density that can be used to extract estimates of the relative strengths of the contributions nf neutrons and protons to the transition:" Quantitative details of these studies can be found in ref. 3

\section{c. Inelastic Scattering, Microscopic Model.}

Of the quite complete comparison of the inelastic data with the predictions of Lee and Kurath ${ }^{1,19}$ we show here only a sample of a few weakly excited states for which theory does only a poor job. We quote from ref. 3 
"In Figure 9 three sets of angular distributions $\left(\pi^{+}, \pi^{+1}\right.$. and $\left.\pi^{-}, \pi^{-1}\right)$ are presented, one each for the states at $3.09 \mathrm{MeV}\left(1 / 2^{+}\right)$, $3.85\left(5 / 2^{+}\right)$, and $8.86 \mathrm{MeV}\left(1 / 2^{-}\right)$. In contrast to the results for the first $9 / 2^{+}$state at $9.5 \mathrm{MeV}$ (see last year's report) the agreement between the calculations and the data is not very good for the $1 / 2^{+}$state at $3.09 \mathrm{MeV}$. According to Lee and Kurath ${ }^{4}$ the $\pi^{-}$transition is dominated by the ( $L=1, S=0$ ) transition density amplitudes, while for $\pi^{+}$the $(L=1, S=0)$ and $(L=1, S=1)$ amplitudes are of comparable magnitude. The excitation of the $(L=1, S=0)$ strength is inhibited for both $\pi^{+}$and $\pi^{-}$by the destructive interference between the amplitudes for $(1 p) \rightarrow(1 d)$ and $(1 p) \rightarrow(2 s)$ excitation. Most of the $(1 p) \rightarrow(2 s)$ amplitude is $1 p_{1 / 2}$ to $2 s_{1 / 2}$ which is almost pure neutron. The differences in the effect of the interference on the $\pi^{+}$and $\pi^{-}$scattering results in quite different theoretical angular distributions. However, the quality of the data is not good enough to say whether the $\pi^{+}$and $\pi^{-}$angular distributiun liave diffcront shapes. Nevertheless, it is apparent that the calculated $\pi^{+}$cross sections are a factor of 3 to 5 smaller than the experimental cross sections. The magnitude of the $\pi^{-}$cross section, however, is consistent with the data. The gross underestimation of the $\pi^{+}$ cross section may be due to the fact that the ground state of ${ }^{13} \mathrm{C}$ as described by the Cohen and Kurath wave functions has too few protons in the $1 \mathrm{p}_{1 / 2}$ orbit. An increase in the $1 \mathrm{p}_{1 / 2}$ proton components in the ground state would increase the $1 \mathrm{p}_{1 / 2}$ to $2 \mathrm{~s}_{1 / 2}$ proton amplitude, decrease the $1 \mathrm{p}_{3 / 2}$ to $1 d_{5 / 2}$ amplitude, and result in less destructive interference for $\pi^{+}$ scattering. Thus the $\pi^{+}$cross section would increase relative to the $\pi^{-}$ cross section. Components in the ${ }^{13} \mathrm{C}$ ground state with two particles in 


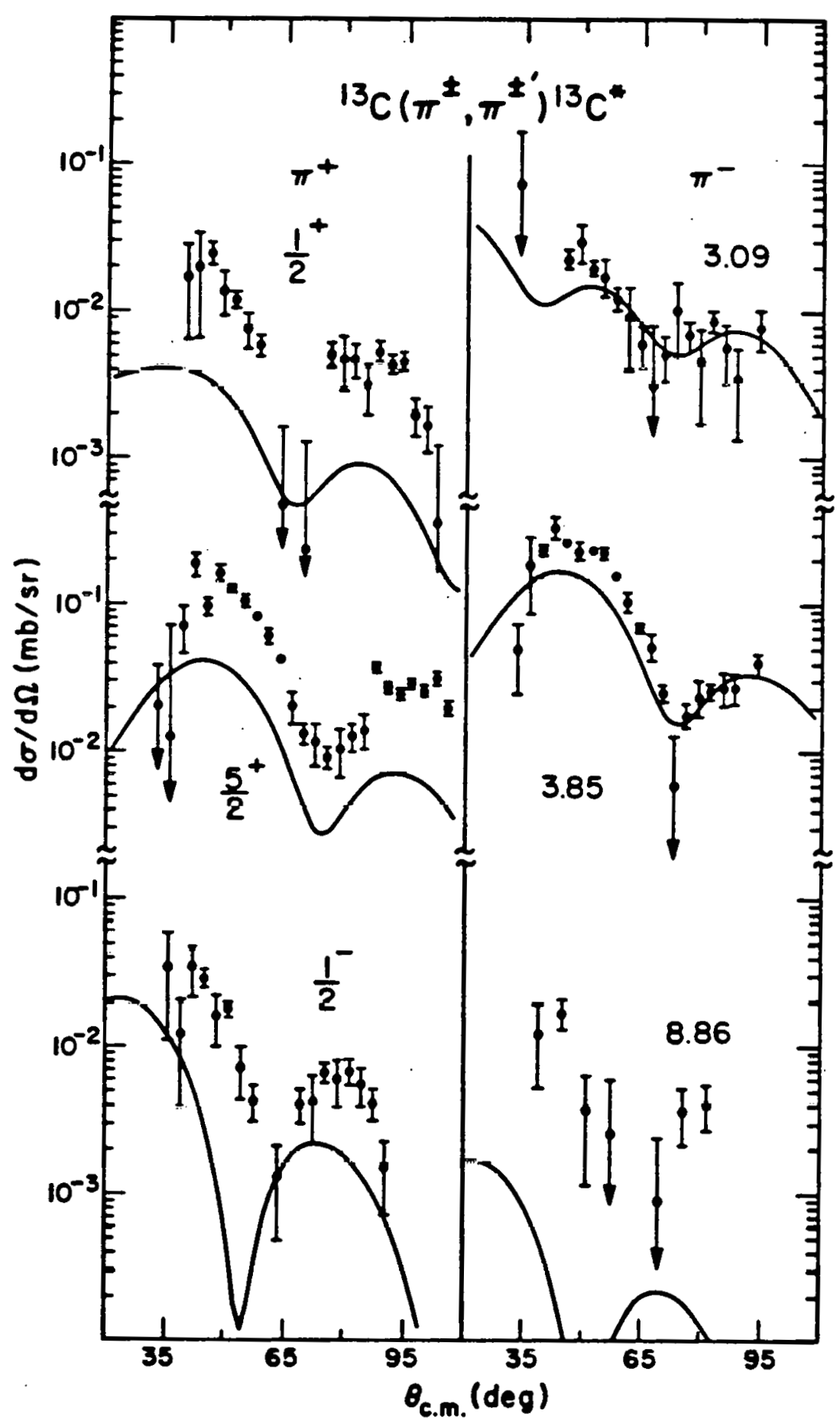

Fig. 9. Inelastic data and microscopic model predictions (ref. 4, 19) for weakly excited states in $13 \mathrm{C}$. 
the (2sld) shell would also increase the cross sections and make the neutron and proton components of the transition density more equal.

The angular distributions in the middle of Fig. 9 are the calculations and the $\pi^{+}$and $\pi^{-}$data for the $5 / 2^{+}$state at $3.85 \mathrm{MeV}$. The calculations ${ }^{4}$ successfully predict the positions of the maxima and minima in the angular distributions but the absolute magnitude is too low for both $\pi^{+}$and $\pi^{-}$. In addition, the calculated ratio $\sigma\left(\pi^{-}\right) / \sigma\left(\pi^{+}\right)$is 4.2 , compared to the experimental value of $\simeq 2$. As for the $1 / 2^{+}$state, the transition to this state is not as neutron dominated as the shell model predictions indicate.

The last set of angular distributions (bottom of Figure 9 ) are for the $1 / 2^{-}$state at $8.86 \mathrm{MeV}$. This transition is very weak and the error bars are quite large. The calculations fail to reproduce both the absolute cross sections and the $\pi^{+} / \pi^{-}$ratio. The predicted $\pi^{+} / \pi^{-}$ratio is about ten, whereas the experimental ratio is between one and two. For $\pi^{+}$the calculated angular distribulion is shifted out of phase with respect to the data. The p-shell model ${ }^{4}$ predicts proton amplitudes much larger than the neutron amplitudes only for the $(L=0, S=1)$ amplitude. For the $(L=2, S=1)$ amplitude the proton to neutron ratio is about two." Thus a letter fit to the data could be obtained with a larger $(L=2, S=1)$ amplitude. of course, the weakness of this transition makes it very likely that twostep processes contribute.

Since the optical potential used by Lee for these calculations does not fit our elastic data very well we plan to repeat the microscopic analysis with our potential sets (see proposal). Nevertheless, since Lee used the same set consistently throughout the p-shell we consider the comparison of the data with these calculations worth publishing and a manuscript is presenlly being propared. 
4. Inelastic Scattering of $\pi^{+}$and $\pi^{-}$from ${ }^{16} 0$ (LAMPF Experiments $\# 369$ and \# 229)

The ${ }^{16} O\left(\pi, \pi^{\prime}\right)$ data from this experiment have now been published 5 . We were very pleased to see that recent $\left(e, e^{\prime}\right)$ and $\left(p, p^{\prime}\right)$ data were analyzed ${ }^{30}$ very successfully when the transition densities were based on the pion work. Within the framework of the DWIA, the proton and electron results are predicted very well by the $1 p-1$ h transition amplitudes for the $4^{-}$states, once the isospin mixing derived from the pion data is properly taken into account. This is a very encouraging result because it implies that consistent and reliable conclusions about nucledr structure can be obtained through comparing pion, proton, and electron excitations of nuclear states. This unification of the three most common intermediate energy probes within the framework of the DWIA is likely to be a fruitful approach in the future.

\section{Other Studies Using Pion Beäms}

The tollowing three investigations were done with Susan SeestromMorris and/or D. B. Holtkamp as participants. Manuscripts have now been submitted for publication and we quote here only the names of all collaborators and the abstract of the paper.

a. Pion Inelastic Scattering to Low Lying States in ${ }^{12} \mathrm{C}$ and ${ }^{40} \mathrm{Ca}$. Christopher L. Morris, Los Alamos Scientific Laboratory, Kenneth C. Boyer, C. Fred Muure, Carol J. Harvey, K. J. Kallianpur, Ingrid B. Moore, and Peter A. Seid1, University of Texas at Austin, Susan J. Seestrom-Morris and D. B. Holtkamp, University of Minnesota, Steven $\mathrm{J}$. Greene and William B.

Cottingame, New Mexico State University. 
Abstract: Pion inelastic scattering at $180 \mathrm{MeV}$ from the low-lying collective states of ${ }^{12} \mathrm{C}$ and ${ }^{40} \mathrm{Ca}$ is analyzed using DWIA calculations with no free parameters and transition densities obtained from electron scattering. These calculations are shown to reproduce the pion scattering data well. No significant $\pi^{+}$vs $\pi^{-}$differences are observed at lowmomentum transfers.

b. Measurements of Isospin Mixing Between the $1^{+}$Doublet in ${ }^{12} \mathrm{C}$ Using Pion Inelastic Scattering. C. L. Morris, R. L. Boudrie and J. Piffaretti, Los Alamos. Scientific Laboratory, W. B. Cottingame, W. J. Braithwaite, S. J. Greene, C. J. Harvey, D. B. Holtkamp, and C. Fred Moore, University of Texas at Austin, S. J. Seestrom-Morris, University of Minnesota.

Abstract: Pion inelastic scattering has been used to extract the offdiagonal charge dependent matrix element $\mathrm{H}_{01}$ between the $\mathrm{J}^{\pi}=1^{+}$doublet of states at $12.71(\mathrm{~T}=\mathrm{U})$ and $15.11(\mathrm{~T}=1) \mathrm{McV}$ in ${ }^{12} \mathrm{C}$. The reported measurements yield a value, $\mathrm{H}_{01}=148 \pm 29 \mathrm{keV}$, in good agreement with recent electromagnetic measurements.

c. Interference Effects in Pion Double Charge Exchange. S. J. Greene, D. B. Holtkamp, W. B. Cottingame, and C. Fred Moore, University of Texas at Austin, G. R. Burleson, New Mexico State University, C. L. Morris, and H. A. Thiessen, Los Alamos Scientific Laboratory, H. T. Fortune, Los Alamos Scientifid Laboratory and University of Pennsylvania. 
Abstract: Pion double charge exchange $\left(\pi^{+}, \pi^{-}\right)$has been measured using targets of ${ }^{16} \mathrm{O}$ and ${ }^{18} \mathrm{O}$ from 80 to $292 \mathrm{MeV}$ at a laboratory angle of $5^{\circ}$. The cross section for the double-analog transition is explained in terms of a direct double-analog amplitude and a two-step non-analog amplitude.

III. Nuclear Structure Studies with Light Ions.

1. Scattering of Polarized Protons from ${ }^{13} \mathrm{C}$. at $547 \mathrm{MeV}$ (LAMPF Expcriment \# 580) Microscopic model calculations 4,19 for inelastic pion scattering on ${ }^{13} \mathrm{C}$ have been very successful for the strongly excited states but failed for several weak transitions. To allow an additional test of the microscopic wave functions we have begun an experiment with polarized protons at HRS. Data were taken for differential cross sections and analyzing powers for many states in ${ }^{13} \mathrm{C}$. The data are now being analyzed using the Love-Francy effective interaction and the transition densities of Lee and Kurath. We are trying to reach a consistent description of our pion and proton data as well as of the electron data from Bates and the 800 MelV proton dat.a nf Blanpied et al. 28

2. Isospin Structure of Nuclear Excitations form a Comparison of the $\left(t, t^{\prime}\right)$ and $\left({ }^{3} \mathrm{He},{ }^{3} \mathrm{He}{ }^{\prime}\right)$ Reactions at Low Energies. During the last year only a few data points had been taken for inelastlc scattering of ${ }^{3} \mathrm{He}$ from ${ }^{13} \mathrm{C}$ and at an energy lower than originally planned because of difficulties with the accelerator. We have now successfully run at the desired energy $\left(E\left({ }^{3} \mathrm{He}\right)=25.4 \mathrm{MeV}\right)$ and both elastic and inelastic data have been taken. In addition we have measured elastic scattering 
cross sections for ${ }^{13} \mathrm{C}+\mathrm{t}$ at $23 \mathrm{MeV}$ when the three-stage mode of the accelerator became operational. Data reduction has been completed.

We have analyzed both elastic data sets $\left({ }^{3} \mathrm{He}+{ }^{13} \mathrm{C}\right.$ and $\left.\mathrm{t}+{ }^{13} \mathrm{C}\right)$ with an optical model and are now applying these potential sets in DWBA and CCBA calculations for the inelastic transitions. Previously we had shown in a CCBA calculation using an optical potential from the literature that the near equality of the $\left(t, t^{\prime}\right)$ and $\left({ }^{3} \mathrm{He},{ }^{3} \mathrm{He} e^{\prime}\right)$ cross sections to the $9 / 2^{+}$state at $9.5 \mathrm{MeV}$ can indeed be understood as mainly due to two-step reaction processes. (This state showed the large asymmetry in the $\left(\pi, \pi^{\prime}\right)$ experiment.) Large $t /{ }^{3} \mathrm{He}$ asymmetries for other states are quite consistent with the pion work. It seems that the $\left(2^{+}\right) \times\left(d_{5 / 2}\right)$ structure of the $9 / 2^{+}$state at $9.5 \mathrm{MeV}$ makes it particularly susceptible to an excitation by a two-step process induced by $t$ and ${ }^{3} \mathrm{He}$. Since nucleon pick-up followed by nucleon stripping is a very urilikely process in $\left(\pi, \pi^{\prime}\right)$ as opposed to $\left(t, t^{\prime}\right)$ and $\left({ }^{3} \mathrm{He},{ }^{3} \mathrm{He}^{\prime}\right)$ the success of the single-step DWIA in reproducing the $9 / 2^{+}$cross sections from $\left(\pi, \pi^{\prime}\right)$ is consistent with the failure of the DWBA for the $\left(t, t^{\prime}\right)$ and $\left({ }^{3} \mathrm{He},{ }^{3} \mathrm{He}{ }^{\prime}\right)$ reactions leading to the same state. Analysis of the data using the new potential sets is in progress. 
IV. Nuclear Structure Studies with Heavy Ions (reported by V. Shkolnik)

1. The ${ }^{16} 0+{ }^{28}$ Si System; Energy Dependence of the Optical Potential, Radial Wave Functions, Parity-Dependence, etc.

In the past year we have shown that our new potential with composite absorption ${ }^{29}$, derived from the 41.23 data for ${ }^{16} \mathrm{O}+{ }^{28}$ Si provides better fits to the elastic scattering than our previous potential over a wide range of incident energies. The article on the elastic scattering for this system which is being readied for publication contains all the details. In brief, the real part of this potential is of $W-S$ shape, as used proviously ${ }^{30}$. The imaginary part is of composite shape with a deep W-S interior and a shallow absorptive peak in the surface 29 . The parameters of this potential are:

$$
\begin{array}{ll}
v_{r}(\mathrm{MeV})=22.10+0.453\left(\mathrm{E}_{1 \mathrm{ab}}-45.0\right) & \mathrm{r}_{\mathrm{r}}=1.323 \mathrm{fm}, \mathrm{a}_{\mathrm{r}}=0.485 \mathrm{fm} \\
w_{v}(\mathrm{MeV})=2 \mathrm{~V}_{\mathrm{r}} & \mathrm{r}_{i}=0.890 \mathrm{fm}, \mathrm{a}_{i}=0.485 \mathrm{fm} \\
w_{d}(\mathrm{MeV})=0.696 \exp \left(0.658 \mathrm{E}_{1 \mathrm{ab}}\right) & \mathrm{r}_{\mathrm{d}}=1.296 \mathrm{fm}, \mathrm{a}_{\mathrm{d}}=0.210 \mathrm{fm} \text { to } 0.310 \mathrm{fm} \\
C_{r}=-0.2081 \exp \left(-0.0631 \mathrm{E}_{1 \mathrm{ab}}\right), & \mathrm{C}_{i}=0, \mathrm{r}_{c}=1.00 \mathrm{fm}
\end{array}
$$

The improvenenls bruught by this torm, compared to the previously used shallow absorptive potential of simple W-S form, are many fold. First, the values of $\chi^{2} / \mathrm{N}$ at the forward angles are improved over the whole energy interval in question; second, the calculated cross sections at the intermediate angles $90^{\circ}<\theta_{\mathrm{cm}}<135^{\circ}$ are now in much hotter agredullut with the data, dud third; the predicted backward rise is now much closer to the $\left[P_{\ell}(\cos \theta)\right]^{2}$ inferred from the data. 
The significance of the new composite form of $W(r)$ in the optical potential goes beyond the listed improvements in the fits and the fact that the region of its application is extended by 3-5 MeV on either side of the previously covered energy interval, $45<\mathrm{E}(1 \mathrm{ab})<63 \mathrm{MeV}$. We have also found strong evidence that the peak in $w(r)$ in the surface and probably part of the interior absorption are due to the coupling to the low-1ying collective states in ${ }^{28} \mathrm{Si}$. Coupled channels calculations were done using the (present) optical potential with reduced real strength and only the interior absorption. Assumed was two-way coupling between the $2^{+}$, $1.78 \mathrm{MeV}$, excited state and the ground state, and a deformation parameter $\beta_{2}=-0.32$. From the radial wave functions obtained from these calculations we selected seven, centered around the grazing partial wave and calculated for each the changes in the optical potential necessary if it were to be used in optical model calculations without coupling. These changes are strongly L-dependent and we have not yet arrived at definite systematic rules on how to incorporate these changes in an L-independent potential. A systematic study of the coupled-channels approach for this system, and of the appropriate ways of adding the contributions to absorption for individual partial waves, should tell us how much of the absorption is due to coupling to the $2^{+}$ excited state.

We have also studied the problem of the ambiguity between the parity dependence and the absorption. Table IV-1 shows for the angular distribution at $E(1 \mathrm{ab})=41.23$ values of $\chi^{2} / \mathrm{N}$ using a grid for $\mathrm{w}_{\mathrm{d}}$ (strength of surface absurption) and $\mathrm{C}_{\mathrm{r}}$ (coefficient of parity dependence). The geometries of the interior and the surface absorption were searched until convergence. This approach focuses on the absorption in the "pocket" since the contribution to the cross sections at the large angles comes from the "pocket", and the parity dependence affects only the large angles. At $41.23 \mathrm{MeV}$ the 
ambiguity has not been resolved yet, possibly due to the uncertainties in the data. (We have found that by introducing a "bump" into the real potential at this energy we can reduce $\chi^{2} / N$ to $\sim 3.5$. The fit is, however, of the same quality as those with $\chi^{2} / N \sim 7$ due to small changes in the fit at the forward angles where the assigned errors are small and influence strongly the average $\chi^{2}$.) All the fits in Table I for $E(1 \mathrm{ab})=41.23 \mathrm{MeV}$ with $X^{2} / N<8$ are equivalent.

In order to gain insight into the details of olastic scallering generated by our new potential we have obtained the wave functions for the partial waves around $\quad \ell_{g r}$ at $E(1 \mathrm{ab})=55.0 \mathrm{MeV}$. Figure 10 shows the moduli $\left|R_{\ell}(r)\right|$ of the radial part of the wave function for each partial wave.

It is readily apparent that we can associate the large peaks at radii between 8 and $10 \mathrm{fm}$ with the combined effect of the Coulomb and centrifugal barriers. The smaller peaks located at radii between 6 and $7 \mathrm{fm}$, apparently are due to. the presence of the "pocket" in the total real potential which is centered at $\sim \mathrm{fm}$. The barrier top is at $\sim 8.2 \mathrm{fm}$. At $\mathrm{E}_{1 \mathrm{ab}}=55 \mathrm{MeV}$ $\left.{ }_{\mathrm{CM}}=35.0 \mathrm{MeV}\right)$ the relatively weak peak for $\ell=25$ is due to "tunneling" since the total potential for $\ell \geq 25$ exceeds $E_{C M}$, at llie barrier radius (Fig. 11). The peaks for $\Omega \leqslant 24$ ean be currelared with the dispersive resonances seen in the phase shifts. The pcaks for partial waves with fncreasingly lower angular momenta become progressively weaker because of increased absorption in the interior. The high sensitivity of the $\chi^{2}$ at large angles to small variations in $W(r)$ in the region of the pocket makes it quite apparent that the large angle cross sections are mainly due to scattering from "inside" the pocket (see ref. 39). 
Table IV.1. Ambiguity between Parity Dependence and Surface Absorption in the Elastic Scattering of ${ }^{16} \mathrm{O}$ from ${ }^{28} \mathrm{Si}$ at $41.23 \mathrm{MeV}$. Values of $\chi^{2} / N$ are tabulated for fits to the angular distribution using a constant real potential and a constant strength for the absorption in the interior. Geometries of the absorption in the interior and in the surface were varied until the search converged.

$\mathrm{E}(1 \mathrm{ab})=41.23 \mathrm{MeV}$

\begin{tabular}{|c|c|c|c|c|c|}
\hline $\mathrm{w}_{\mathrm{d}}(\mathrm{MeV})$ & -0.0050 & -0.0100 & -0.0155 & -0.0200 & -0.0250 \\
\hline 0.350 & 95.0 & 73.2 & 21.9 & 12.4 & 35.7 \\
\hline 0.700 & 26.6 & 14.3 & 6.5 & 5.8 & 7.2 \\
\hline 1.050 & 14.5 & 8.6 & 6.1 & 7.4 & 9.9 \\
\hline 1.400 & 12.3 & 8.1 & 7.0 & 9.4 & 12.3 \\
\hline 1.750 & 11.8 & 8.5 & 8.6 & 11.5 & 14.9 \\
\hline 2.100 & 12.8 & 9.6 & 10.3 & 13.3 & 14.6 \\
\hline 2.450 & 13.8 & 11.1 & 11.8 & 14.2 & 14.7 \\
\hline
\end{tabular}



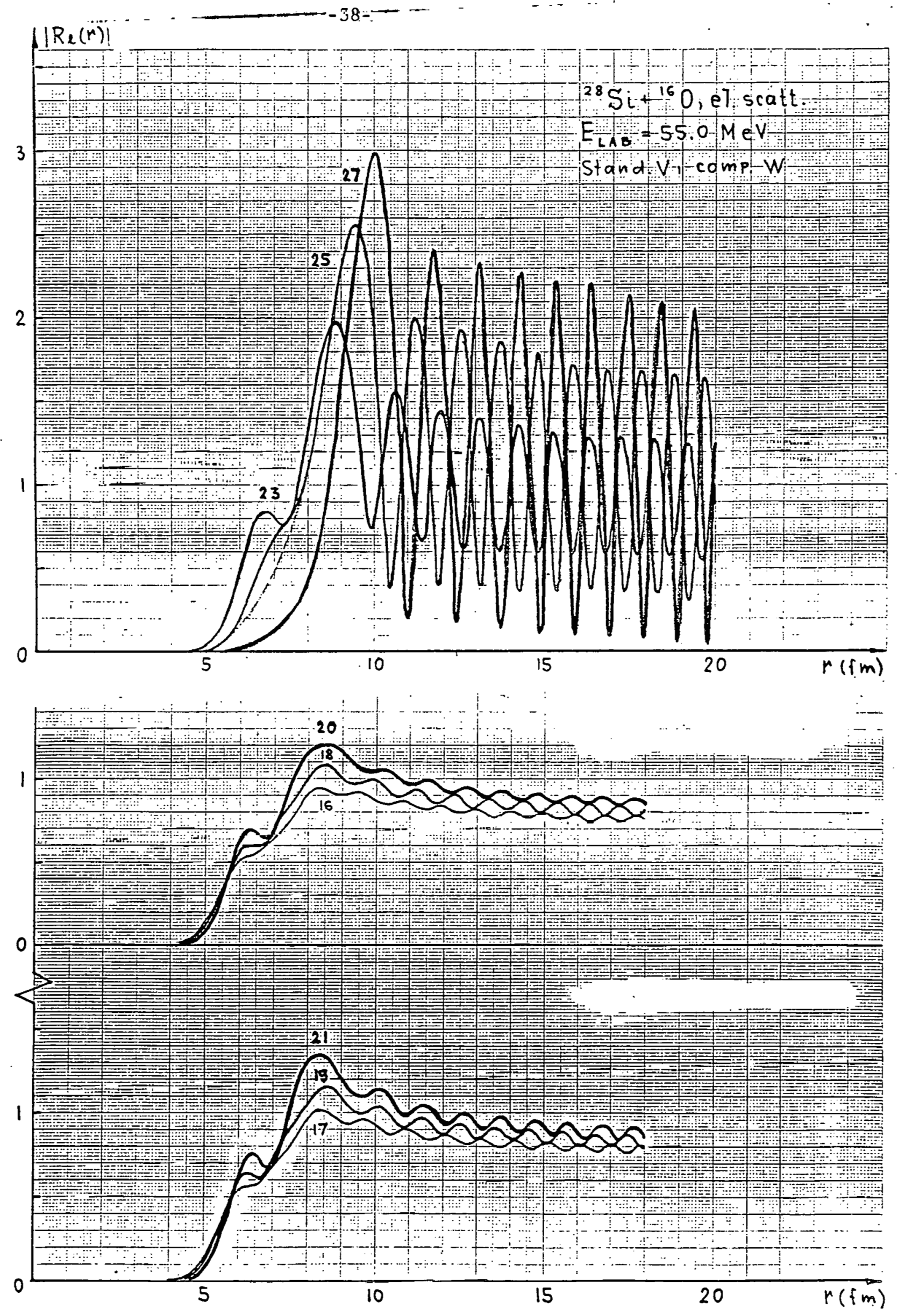

Fig. 10. Moduli of radial wave functions $\left|R_{\ell}(r)\right|$. 
We will now discuss a property of the surface-transparent potentials that may be used as a guide in estimating the real well depth. In several studies (ref. 29-35) of elastic scattering of $40-60 \mathrm{MeV}{ }^{16} \mathrm{O}$ on eight targets ranging from ${ }^{16} 0$ to ${ }^{40} \mathrm{Ca}$ it was found that close fits to the data could be obtained only by use of surface transparent potentials. The real and imaginary strengths of these potentials generally increase with energy. Specifically the real well depth increases linearly with energy in this energy range and is deep enough to generate a "pocket" in the total potential $\quad V_{\text {tot }}(r, l)$ at least for partial waves around and below the grazing partial wave (Fig. 11), where

$$
V_{\text {tot }}(r, \ell)=-V_{0} f(r)+\frac{z_{1} z_{2} e^{2}}{r}+\frac{\hbar^{2}}{2 \mu r^{2}} \ell(\ell+1) . \quad \text { Eq. IV.1.1. }
$$

The pocket causes a discontinuity (as a function of $l$ ) in the distance of closest approach (e.g. at $\ell \approx 24$ for ${ }^{16} 0+{ }^{28} \mathrm{Si}$ at $35 \mathrm{MeV}$ (c.m.)). Assuming analytic continuity for $\ell$ we can define an angular momentum $\ell_{c}$ by the condition that $E_{c . m}$. is tangential to $V_{\text {tot }}$ a.t. the barrier top radius $r_{b}=\tilde{r}_{b}\left(A_{1}^{1 / 3}+A_{2}^{1 / 2}\right.$ ). This condition can be expressed by two equations

$$
\left.\frac{d V_{\text {tot }}}{d r}\right|_{r_{b}, l_{c}}=n \text { and } V_{\text {tot }}\left(r_{b}, l_{c}\right)=E_{c . m .} \quad \text { Eq. IV.1.2. }
$$

We find that $\ell_{c}$ is very close to the grazing angular momentum $\ell_{g r}$ derived from the quarter-point angle $\left(\ell_{g r}=\eta \cot \frac{\theta_{1 / 4}}{2}\right)$ and also to the critical angular momentum $\ell_{\mathrm{cr}}$ from the S-matrix elements, $\left(\left|\mathrm{s}_{\ell_{\mathrm{cr}}}\right|^{2}=0.5\right)$.

Since the reduced radius, $\widetilde{r}_{b}$, appears to be quite independent of target mass and incident energy eq. IV.1.2 may be used as a guide to estimate the real well depth simply from $\ell_{g r}$ (derived from $\theta_{1 / 4}$ ) provided that $\ell_{\mathrm{gr}} \approx \ell_{c}$. We have used this procedure for the analysis of ${ }^{16} \mathrm{O}+{ }^{40} \mathrm{Ca}$ data with encouraging results. 


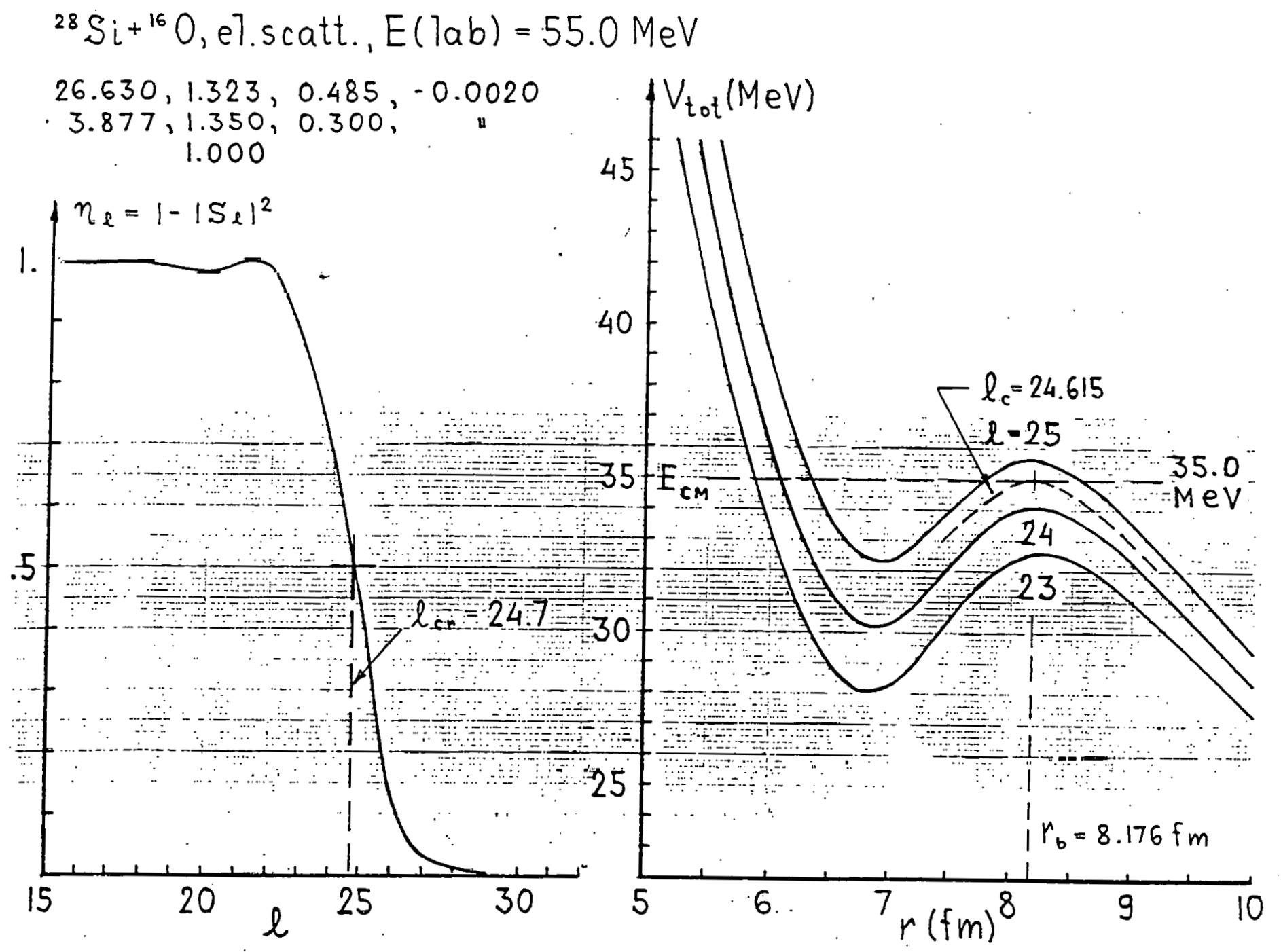

Fig. 11. Absorption coefficient $\eta_{\ell}=1-\left|s_{\ell}\right|^{2}$ as a function of $\ell$ (1eft) and the total potential energy $V_{\text {tot }}(r, l)$ for $l=23,24$, and 25 (right). 
2. The ${ }^{16} \mathrm{O}+{ }^{40} \mathrm{Ca}$ Potential Between 37 and $65 \mathrm{MeV}$

The data of Kubono et al (ref. 35) for the elastic scattering of ${ }^{16} \mathrm{O}$ from ${ }^{40} \mathrm{Ca}$ consist of one complete angular distribution at $\mathrm{E}(1 \mathrm{ab})=$ $50.0 \mathrm{MeV}$ and a $180^{\circ}$ excitation function taken between $\mathrm{E}(\mathrm{lab})=37.29 \mathrm{MeV}$ and $70.25 \mathrm{MeV}$. Kubono's fit to the angular distribution (Fig. 12) is very good up to $\theta_{\mathrm{cm}} \sim 120^{\circ}$ but contains one peak more than the data at larger angles. Thus the fit is out of phase with the data at some angles. No fit to the $180^{\circ}$ excitation function was presented in ref. 35. Angular distributions measured nver limited ranges of angles exist at the following energies: $E(l a b)=38.0,40.0$ and $42.0 \mathrm{MeV}$ (ref. 36), at $E(l a b)=40.0 \mathrm{MeV}$ (ref. 37), at $E(1 \mathrm{ab})=55.6$ and $60.0 \mathrm{MeV}$ (ref. 38), and at $E(1 \mathrm{ab})=74.4$ (ref. 40).

We have obtained fits to the $50.0 \mathrm{MeV}$ angular distribution using surface-transparent and parity-dependent optical potentials with real parts of simple and composite geometries and imaginary parts of composite geometry. A representative fit is shown in Fig. 13. These optical potentials employ parity dependence in their real and imaginary parts. The strength of the parity dependence is only $1 / 4$ to $1 / 3$ of what was used with the potential with composite $W(r)$ for ${ }^{16} \mathrm{O}+{ }^{28} \mathrm{Si}$ at $\mathrm{E}(1 \mathrm{ab})=50.0 \mathrm{MeV}$. The fit shown in Fig. 13 is perfect at large angles but not quite adequate at the forward angles. We hope that the use of a deeper real part and especially the use of a deeper interior absorption, like in the case of ${ }^{16} \mathrm{O}+{ }^{28} \mathrm{Si}$, will provide a better fit. Yet, by using the geometry parameters

$$
\begin{aligned}
& r_{r}=1.431 \mathrm{fm}, \quad a_{r}=0.350 \mathrm{fm}, \quad r_{c 011}=1.200 \mathrm{fm} \\
& r_{i}=1.427 \mathrm{fm}, \quad a_{i}=0.161 \mathrm{fm}, \quad r_{d}=1.731 \mathrm{fm}, \quad a_{d}=0.080 \mathrm{fm},
\end{aligned}
$$




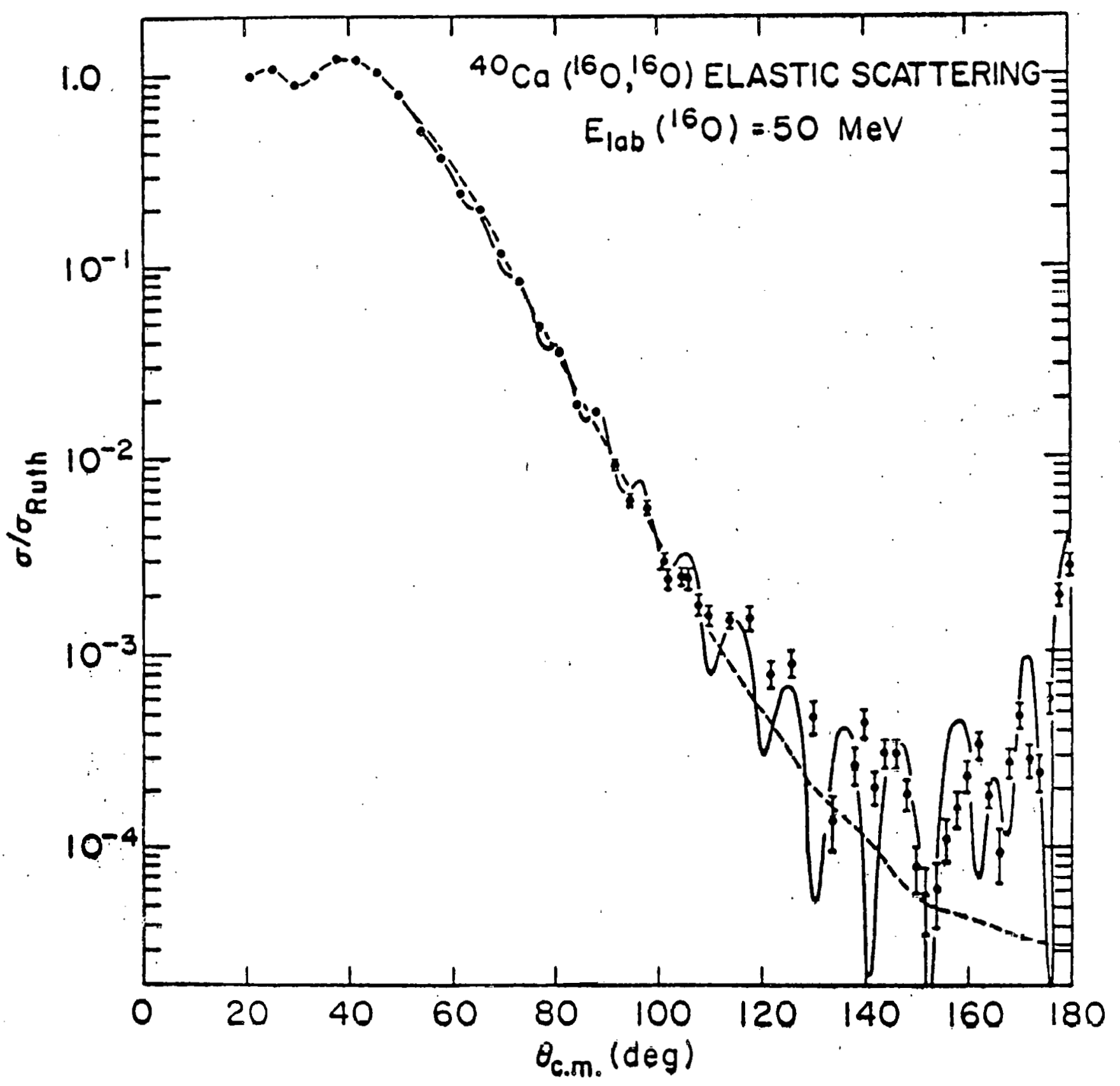

Fig. 12. Data and fit of Kubono, et al. (ref. 35). 


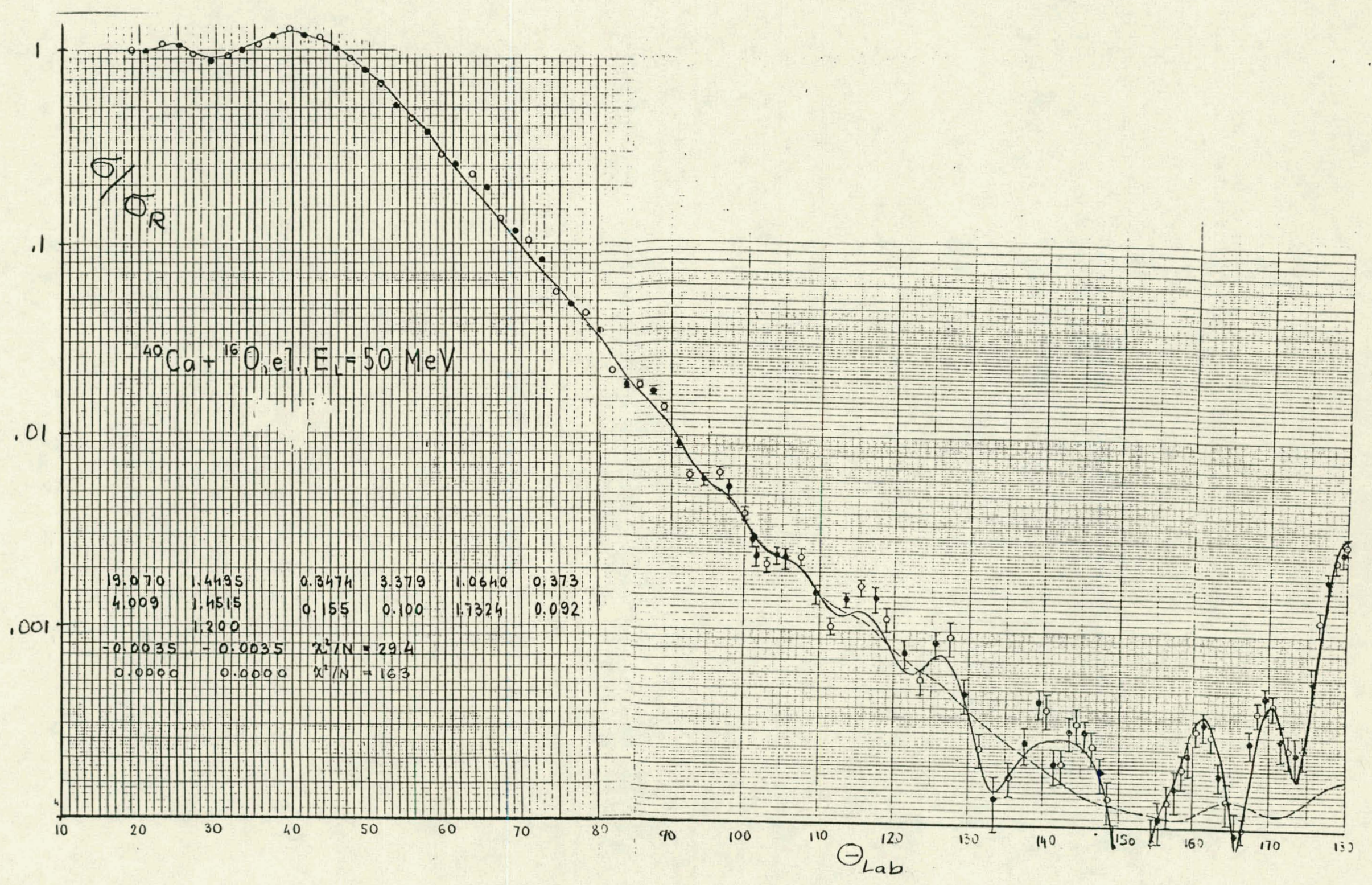

Fig. 13. Fit to data of ref. 35 from this work. 
and the energy dependence of the real and interior imaginary strengths $\mathrm{V}_{\mathrm{r}}$ and $\mathrm{W}_{\mathrm{V}}$ as shown in Fig. 14, constant absorption in the surface $\mathrm{w}_{\mathrm{d}}=0.136 \mathrm{MeV}$, and constant parity dependence $C_{r}=C_{i}=-0.0023$, we obtained a good fit over a substantial portion of the $(180 \pm 5.7)^{\circ}$ excitation function (Fig. 15). The dashed line in Fig. 14 is a prediction for $V_{0}\left(E_{c m}\right)$ using the method (Eqs. IV.1.1) outlined in the previous section. 


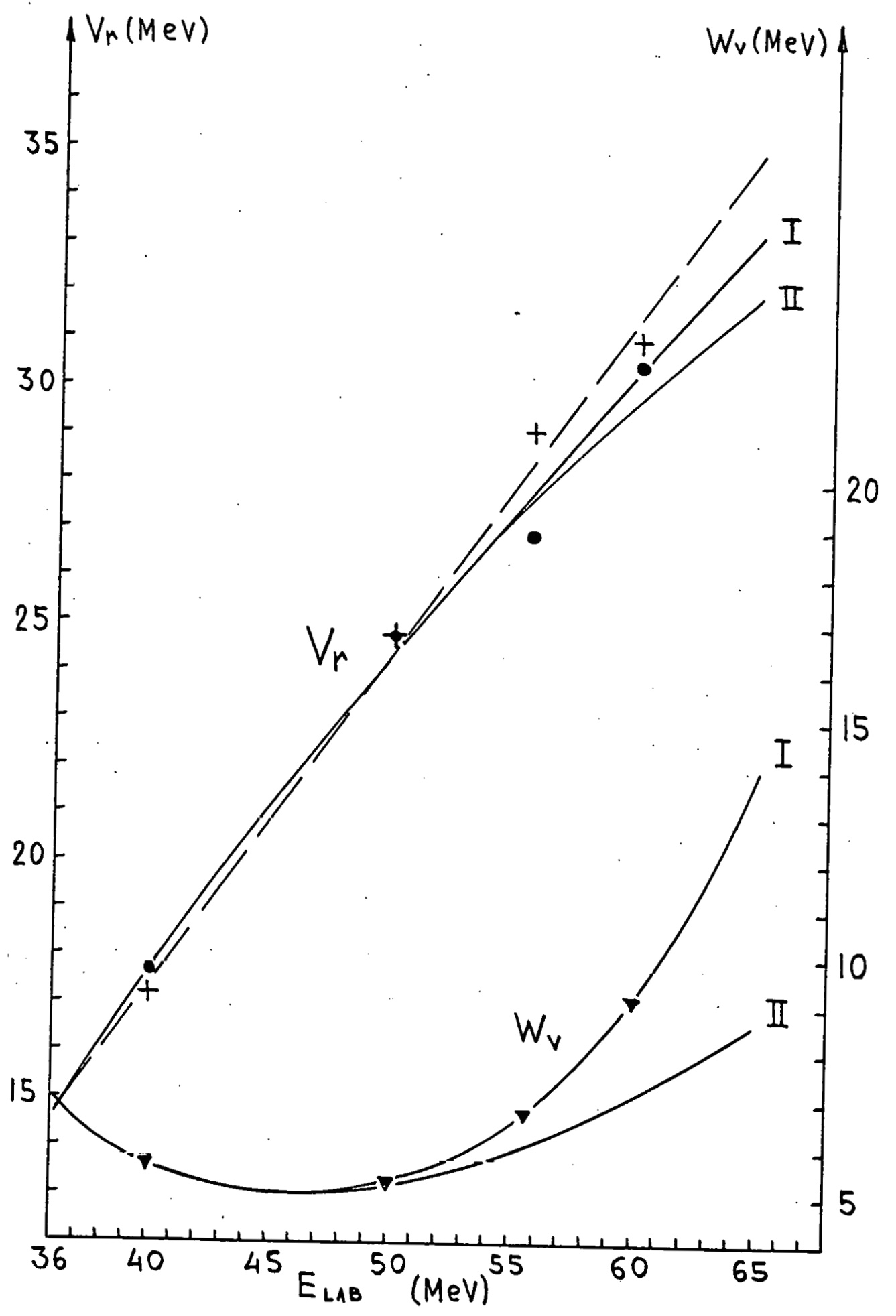

Fig. 14. Energy dependence of real and imaginary depths used in fits to the $160+40 \mathrm{Ca} 180^{\circ}$ excitation function shown in Fig. 15 . 


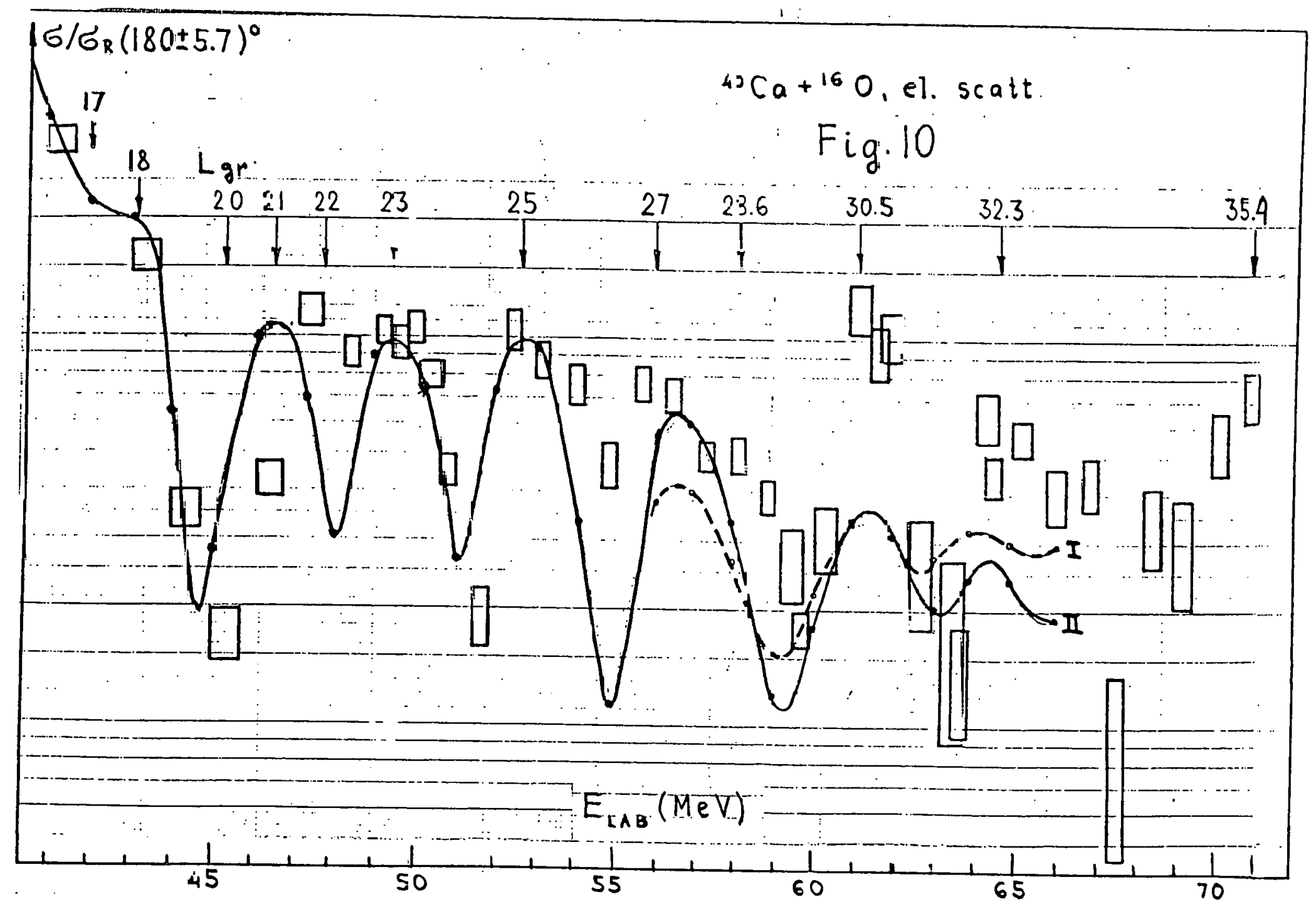

Fig. 15. ${ }^{16} 0+{ }^{40} \mathrm{C} c$ excitation function (ref. 35) and fits Irom this work. 
References

1. F. Ajzenberg-Selove, Nucl. Phys. A268 (1976) 1 and to be published.

2. C. L. Morris et al., Phys. Lett. 86B (1979) 31.

3. S. J. Seestrom-Morris, Ph.D. Thesis, University of Minnesota, 1981.

4. T.-S. H. Lee and D. Kurath, Phys. Rev. C22 (1980) 1670.

5. D. B. Holtkamp et al., Phys. Rev. Lett. 43 (1979) 1091.

6. S. Cohen and D. Kurath, Nucl. Phys. 73 (1967) 1.

7. G. Kaschl, G. Mairle, H. Mackh, D. Hartwig, and U. Schwinn, Nucl. Phys. A178, (1971) 275 .

8. H. Crannel et al., Nucl. Phys. A278 (1977) 253, and D. Kurath, private communication.

9. T.-S. H. Lee and R. D. Lawson, Phys. Rev. C21 (1980) 679.

10. T. Engeland and P. J. Ellis, Nucl. Phys. Al44 (1970) 161 and Al81 (1972) 368.

11. S. Lie, Nucl. Phys. Al81 (1972) 517.

12. N. F. Mangelson, B. G. Harvey, and N. K. Glendenning, Nucl. Phys. All7 (1968) 161 and references therein. H. T. Fortune, private communication.

13. D. Kurath, private communication. In this calculation one particle in the (sd) shell is coupled to all the p-shcll states of ref. 6 to generate the spectrum of negative parity states.

14. D. J. Millener and D. Kurath, Nucl. Phys. A255 (1975) 315.

15. D. S. Koltun, in Advances in Nuclear Science, Vol. 3, Edited by M. Baranger and E. Vogt, p. 71 (Plenum Press 1969).

16. E. R. Siciliano and G. E. Walker, to be published.

17. C. Fred Moore, et al., Phys. Lett. 80B (1978) 38. 
18. W. B. Cottingame, et al., Bul1. Am. Phys. Soc. 24, 821 (1979), and to be published.

19. T. S. H. Lee and D. Kurath, Phys. Rev. C21, 293 (1980).

20. R. A. Eisenstein and G. A. Miller, Comp. Phys. Comm. 11, 95 (1976).

21. A. M. Lane, Rev. Mod. Phys. 32, 519 (1960).

22. D. Dehnhard, et al., Phys. Rev. Lett. $\underline{43}$ (1979) 1961.

23. R. S. Hicks, et al., Proceedings of the Workshop on Nuclear Structure with Intermediate Energy Probes, Los Alamos, ed. by H. A. Thiessen, $292(1980)$.

24. J. Dubach, et a1., to be published.

25. E. H. Auerbach, D. M. Fleming, and M. M. Stcrnheim, Phys. Rev. 162 (1967) 1683.

26. W. B. Cottingame and D. B. Holtkamp, Phys. Rev. Lett. $\underline{45}$ (1980) 1828.

27. F. Petrovich and W. G. Love, Invited Talk, Int. Conf. on Nuclear Physics, Berkeley, Cali,fornia, Aug. 24-30, 1980.

28. G. S. Blanpied, Ph.D. Thesis, U. of Texas, 1978.

29. V. Shkolnik et al., Proc. of the Intern. Conf. on Nuclear Physics, Berkeley, 1980, p. 588 .

30. D. Dehnhard et al., Phys. Rev. Lett. 40 (1978) 1549; Erratum, Phys. Ref. Lett. $\underline{42}$ (1979) 1574.

31. J. V. Maher et al., Phys. Rev, 188 (1969) 1665.

32. K. Siwek-Wilczynska et a1., Nuc1. Phys. A229 (1974) 461.

33. H. T. Fortunc et ai., f'hys. Rev. C20 (1979) 638.

34. V. Shkolnik et al., Phys. Lett. 74B (1978) 195.

35. S. Kubono et al., Phys. Lett. 81B (1979) 140. 
36. J. S. Eck et al., Phys. Rev. 186 (1969) 1132.

37. K. 0. Groenevelt et al., Phys. Rev. C6 (1972) 805.

38. S. Kubono, unpublished.

39. D. M. Brink and N. Takigawa, Nucl. Phys. A279 (1977) 159.

40. S. E. Vigdor et al., Phys. Rev. C20 (1979) 2147. 


\section{Publications, Abstracts, Invited Talks}

A. Pub1ications

D. B. Holtkamp, W. J. Braithwaite, W. Cottingame, S. J. Greene,

R. J. Joseph, C. Fred Moore, C. L. Morris, J. Piffaretti, E. R. Siciliano, H. A. Thiessen, and D. Dehnhard, "Isospin Mixing of $4^{-}$Particle-Hole States in 160," Phys. Rev. Letters 45, 420-423 (1980).

W. B. Cottingame, and D. B. Holtkamp, "Energy Dependence of Pion Elastic Scattering from Nuclei Across the $(3,3)$ Resonance," Phys. Rev. Letters, published Phys. Rev. Letters $\underline{45}, 1828$ (1980).

G. R. Burleson, G. S. Blanpied, G. H. Daw, A. J. Viescas, C. L. Morris,

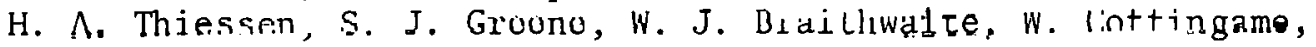

D. B. Hulckamp, L. Fred Moore, and I. B. Moore, "Isospin Quintets in the Ip and s-d Shells," Phys. Rev. C, 22, 1180-1183 (1980).

C. L. Morris, H. A. Thiessen, W. J. Braithwaite, W. B. Cottingame, S. J. Greene, D. B. Holtkamp, I. B. Moure, C. Fred Moore, G. R. Burlcson, G. S. Blanpied, G. H. Daw, and A. J. Viescas, "Observation of a Double Isobaric Analog State in the Reaction ${ }^{209} \mathrm{Bi}\left(\pi^{+}, \pi^{-}\right){ }^{209} \mathrm{At}$," Phys. Rev. Letters 45, 1233-1234 (1980).

B. Papers in press or submitted for publication

Christopher L. Morris, Kenneth G. Boyer, C. Fred Moore, Carol J. Harvey, K. J. Kallianpur, Ingrid B. Moore, Peter A. Seid1, Susan J. SeestromMorris, D. B. Holtkamp, Steven J. Greene, and William B. Cottingame, "Pion Inelastic Scattering to Low-Lying States in ${ }^{12 \mathrm{C}}$ and ${ }^{40} \mathrm{Ca}, "$ Phys. Rev. C., to be published.

C. L. Prisis, R. L. Boudr1e, J. Piffaretti, W. B. Cottingame, W. J. Braithwaite, S. J. Greene, C. .T. Harvey, C. Frcd Mooie, D. B. Holtkamp, S. J. Tripp, "Measurements of Isospin Mixing Between the $1^{+}$Doublet in 12 Using Pion Inelastic Scattering, "submitted for pub1..

S. J. Seestrom-Morris n. Dehnhard, D. B. Ilu1thinly, and C. L. Morris, "Identification of $\Delta S=1$ Transitions by Measurement of Pion Inelastic Excitation Functions," submitted for publ..

\section{Papers in progress}

D. B. Holtkamp, S. J. Seestrom-Morris, S. Chakravarti, D. Dehnhard, H. W. Baer, C. L. Morris, S. J. Greene, and C. J. Harvey, "Strong Cancellations of Neutron and Proton Transition Amplitudes in Pion Inelastic Scattering from ${ }^{14} \mathrm{C} . "$

S. J. Seestrom-Morris, D. Dehnhard, et a1., "Structure of ${ }^{13} \mathrm{C}$ Studied 
by Pion Scattering Near the $(3,3)$ Resonance."

D. Contributions to Conferences and Workshops

S. J. Tripp, D. Dehnhard, M. A. Franey, G. S. Kyle, C. L. Morris, R. L. Boudrie, J. Piffaretti, and $H$. A. Thiessen, "Energy Dependence of Inelastic Pion Scattering from ${ }^{13} \mathrm{C}$, " Proceedings of the LAMPF Workshop on Nuclear Structure with Intermediate Energy Probes, Los Alamos, New Mexico, LASL Report no. LA-8303-C, p. 328 (1980).

W. J. Braithwaite, S. J. Greene, I. B. Moore, W. B. Cottingame, D. B. Holtkamp, C. Fred Moore, C. L. Morris, H. A. Thiessen, G. R. Burleson, G. S. Blanpied, "Observation of the Double Isobaric Analog State in the Reaction $209_{\mathrm{Bi}}\left(\pi^{+}, \pi^{-}\right){ }^{209} \mathrm{At} . "$ Proceedings of the LAMPF Workshop on Nuclear Structure with Intermediate Energy Probes, Los Alamos, New Mexico, LASL Report no. LA-8303-C, 331 (1980).

S. J. Greene, W. J. Braithwaite, D. B. Holtkamp, W. B. Cottingame, C. Fred Moore, G. R. Burleson, G. S. Blanpied, C. L. Morris, H. A. Thiessen, "Pion Double Charge Exchange - Recent Data," Proceedings of the LAMPF Workshop on Nuclear Structure with Intermediate Energy Probes, Los Alamos, New Mexico, LASL Report no. LA-8303-C, 430 (1980).

D. B. Holtkamp, and W. B Cottingame, "Global Fits of Pion Elastic Scattering from ${ }^{12} \mathrm{C}$ and $16 \mathrm{O}$ across the $(3,3)$ Resonance," Proceedings of the LAMPF Workshop on Nuclear Structure with Intermediate Energy Probes, Los Alamos, New Mexico, LASL Report no. LA-8303-C, 439 (1980).

D. B. Holtkamp, and W. B. Cottingame, "Global Fits of Pion Elastic Scattering," Proceedings of the International Conference on Nuclear Physics, Berkeley, California, 699 (1980).

W. B. Cottingame, W. J. Braithwaite, R. J. Joseph, D. B. Holtkamp, S. J. Greene, C. F. Moore, R. L. Boudrie, C. L. Morris, J. Piffaretti, H. A. Thiessen, "Measuring Isospin Mixing in Nuclei Using $\pi \pm$ Inelastic Scattering," Proceedings of the International Conference on Nuclear Physics, Berkeley, California, 706 (1980).

K. G. Boyer, W. J. Braithwaite, W. Cottingame, S. J. Greene, C. J. Harvey, D. B. Holtkamp, L. E. Smith, C. F. Moore, C. L. Morris, H. A. Thiessen, C. S. Blanpied, G. R. Burleson, J. Davis, J. S. McCarthy, R. C. Minehart, C. A. Goulding, "Comparison of $\pi^{+}$and $\pi^{-}$Inelastic Scattering From Low-Lying Collective States of $40,42,44,48 \mathrm{Ca}$ and $54 \mathrm{Fe}, "$ Proceedings of the International Conference on Nuclear Physics, Berkeley, California, 695 (1980).

V. Shkolnik, M. A. Franey, and D. Dehnhard, "A Composite Absorptive Potential for $160+28 \mathrm{Si}$ Elastic Scattering", Proceedings of the International Conference on Nuclear Physics, Berkeley, California 1980, page 588. 


\section{E. Abstracts}

D. B. Holtkamp, C. L. Morris, W. B. Cottingame, S. J. Greene, W. J. Braithwaite, C. J. Harvey, C. F. Moore, I. B. Moore, P. A. Seidl, "A Study of the $1^{+}$States of ${ }^{12} \mathrm{C}$ with Pion Inelastic Scattering: Angular Distributions and Excitation Function," Bull. Am. Phys. Soc. 25, 730-731 (1980).

C. Fred Moore, W. J. Braithwaite, C. J. Harvey, K. Kallianpur,

I. B. Moore, P. A. Seidl, D. B. Holtkamp, W. B. Cottingame, S. J. Greene, C. L. Morris, "A Comparison of Inelastic Pion Scattering from Self Conjugate Nuclei ${ }^{40} \mathrm{Ca}$ and ${ }^{12} \mathrm{C}, " \mathrm{Bull}$. Am. Phys. Soc. 25, 731 (1980).

R. L. Boudrie, J. F. Amann, G. C. Idzorek, S. J. Seestrom-Morris, D. B. Holtkamp, M. A. Franey, D. Dehnhard, C. Goulding, "Scattering of $547 \mathrm{MeV}$ Polarized Protenns from! ".3 $\mathrm{C}^{1 \mathrm{Bu}} 1$. Am. Phys. Soc., Lo be published.

S. J. Seestrom-Morris, D. Dehnhard, M. A. Franey, G. S. Kyle, C. L. Morris, R. L. Boudrie, J. Piffaretti, and H. A. Thiessen" DWIA Analysis of Elastic and Inelastic Scattering of Pions from ${ }^{13} \mathrm{C}, "$ Bull. Am. Phys. Soc., to be published.

D. B. Holtkamp, S. J. Seestrom-Morris, D. Dehnhard, H. W. Baer, C. L. Morris, S. J. Greene, and C. J. Harvey, Bul1. Am. Phys. Soc., to be published.

\section{F. Invited Ta1ks}

D. Dehnhard

Nuclcar Seminas at the University of Pcnnsylvania.

Invitod eulk on "Unialural Par1ty Excitations in Intermediate Energy

Pion and Proton Scattering," given at the Minneapnlis Meeting of thc Division of Nuclear Physics, Oct. 9-11, 1980, Bul1. Am. Phys. Soc. 25 (1980) 732, paper CA3.

G. Advanced Degree Granted

Susan J. Seestrom-Morris, Ph.D. March '81. Thesis title: "The Structure of ${ }^{13} \mathrm{C}$ Studied by Pion Scattering Near the $(3,3)$ Resonancc." 
VI. Distribution of Effort of Principal Investigator

1979-80. Acad. Year (June 1-15, 1980) 45\%

$1980 \quad$ Summer (July 1 - Sept. 15, 1980) 100\%

1980-81 Acad. Year (Sept. 16-Dec. 15, 1980) 90\%

1980-81 Acad. Year (Dec. 16 - present) 50\%

Expected contribution from present to May $31 \quad 50 \%$ 